\title{
A DYNAMICAL ANALYSIS OF THE H II GALAXY II ZWICKY 33 AND ITS LOW SURFACE BRIGHTNESS COMPANION
}

\author{
FABIAN WALTER ${ }^{1}$ \\ National Radio Astronomy Observatory, P.O. Box O, Socorro, New Mexico 87801, and Department of Physics and Astronomy, \\ University of New Mexico, Albuquerque, New Mexico 87131 \\ Electronic mail: fwalter@nrao.edu \\ ELIAS BRINKS \\ National Radio Astronomy Observatory, P.O. Box O, Socorro, New Mexico 87801, and Departamento de Astronomía, \\ Apartado Postal 144, Guanajuato, Gto. 36000, Mexico \\ Electronic mail: ebrinks@nrao.edu \\ NEB DURIC \\ Department of Physics and Astronomy, University of New Mexico, Albuquerque, New Mexico 87131 \\ Electronic mail: duric@deneb.phys.unm.edu \\ ULRICH KLEIN \\ Radioastronomisches Institut der Universität, Auf dem Hügel 71, D-53121 Bonn, Germany \\ Electronic mail: uklein@astro.uni-bonn.de \\ Received 1996 November 25, revised 1997 February 24
}

\begin{abstract}
We present radio and optical observations and an analysis of the dynamics of the $\mathrm{H}$ II galaxy II Zw 33 and its Low Surface Brightness companion. Both galaxies were observed in the radio with the VLA in its B- and $\mathrm{C}$-array configurations and in the optical ( $B$-band) with the Kitt Peak 4-m telescope. II Zw 33 is an example of an H II galaxy, i.e., a dwarf galaxy in which violent star formation is taking place. The absolute blue magnitude of II Zw 33 is $M_{B}=-18.37 \mathrm{mag}$; the amount of observed $\mathrm{H} \mathrm{I}$ mass is $M_{\mathrm{HI}}=1.01 \times 10^{9} \mathrm{M}_{\odot}$ leading to a $\mathrm{H} \mathrm{I}$ mass to blue-light ratio of $M_{\mathrm{HI}} / L_{B}=0.31\left(\mathrm{M}_{\odot} / \mathrm{L}_{\mathrm{B} \odot}\right)$. The companion, II $\mathrm{Zw} 33 \mathrm{~B}$, shows hardly any optical emission in the $B$-band (absolute blue magnitude $M_{B}=-15.82 \mathrm{mag}$ ) and is considered to be a low surface brightness galaxy. It has, however, almost the same size and $\mathrm{HI}$ mass $\left(M_{\mathrm{HI}}=0.59 \times 10^{9} \mathrm{M}_{\odot}\right)$ as the parent galaxy II $\mathrm{ZW} 33$, resulting in an $\mathrm{H} \mathrm{I}$ mass to blue-light ratio of $M_{\mathrm{H} \text { I }} / L_{B}=1.83\left(\mathrm{M}_{\odot} / \mathrm{L}_{\mathrm{B} \odot}\right)$. A dynamical study shows that no dark matter is needed to within the last measured point of the rotation curve $(r<6.6 \mathrm{kpc})$ to explain the observed rotation curve of II $\mathrm{Zw} 33$. This is a major difference to other dwarf galaxies studied so far which seem to be dark matter dominated throughout. The total (dynamical) mass for II $\mathrm{Zw} 33$ is $M_{\text {tot }}=5.1 \times 10^{9} \mathrm{M}_{\odot}$. As it turns out, dark matter is not needed either to explain the rotation curve of the companion (total mass: $M_{\text {tot }}=1.2 \times 10^{9} \mathrm{M}_{\odot}$ ). There is a striking difference between the shape of the rotation curves of II $\mathrm{Zw} 33$ and its companion which is attributed to the presence of stars in the former. In the course of our modelling we derive $M_{*} / L_{B}=1\left(\mathrm{M}_{\odot} / \mathrm{L}_{\mathrm{B} \odot}\right)$ for both objects. If we assume the system to be bound, however, this requires that at least $44 \times 10^{9} \mathrm{M}_{\odot}$ of mass must be present in the system, presumably mostly in the form of dark matter surrounding the objects. (C) 1997 American Astronomical Society. [S0004-6256(97)00906-0]
\end{abstract}

\section{INTRODUCTION}

Dwarf galaxies are unique objects and play a vital role in our attempts to understand galaxy formation, galaxy evolution, star formation, and the problem of Dark Matter, to name but a few. This is mainly a consequence of dwarf galaxies being smaller and simpler systems compared to normal galaxies (e.g., they don't have a bulge and are too small to

${ }^{1}$ Current address: Radioastronomisches Institut der Universität, Auf dem Hügel 71, D-53121 Bonn, Germany. sustain a spiral density wave); they are therefore almost ideal laboratories for the studies listed above.

Dwarf galaxies come in three main types, dwarf Ellipticals (dE), dwarf Spheroidals (dSph), and dwarf Irregulars (dIrr). It has been known for some 25 years now that star formation (SF) in dIrrs occurs in bursts. This bursting phenomenon results in an $\mathrm{H}$ II galaxy (often also referred to as Blue Compact Dwarf (BCD) galaxy or extragalactic $\mathrm{H}$ II region), II $\mathrm{Zw} 33$ being one of them. It is not at all clear, though, what triggers SF in these objects. Since spiral density waves are obviously not present in dwarf galaxies, a different process for SF must dominate. Mueller \& Arnett 
(1976) proposed that SF can be "self-propagating" via shock waves created by SN explosions due to the most massive stars created in each SF region. Gerola \& Seiden (1978) expanded this model, treating local SF as a stochastic process which they called "self-propagating stochastic star formation" (SPSSF). A remaining problem, though, is that neither description explains what causes the very first burst of SF. Nor is it clear in Gerola \& Seiden's approach to what the spontaneous SF probability is related. To address these issues, another, more recent proposition was put forward which is that interactions are responsible for the bursts of star formation in $\mathrm{H}$ II galaxies. From work by Campos-Aguilar et al. (1993) and Telles \& Terlevich (1995) it has become clear that these interactions are not with bright galaxies. Rather, as was suggested by Brinks (1990) and confirmed by Taylor et al. (1993) and Taylor et al. (1995, 1996), interactions with optically inconspicious objects, such as dwarf companions, are the likely trigger for the burst of star formation. The idea being that most of the gas in a galaxy is below a critical density for SF and that an interaction with a companion increases the surface-density in the dwarf galaxy locally until it reaches a critical density (Noguchi 1988).

An example of a dwarf galaxy which might have been triggered into action is the dIrr galaxy II $\mathrm{Zw} 33$ (also known as Mrk 1094). Brinks (1990) using the $\mathrm{VLA}^{2}$ in its B-array configuration discovered a companion $\mathrm{H}$ I cloud within 5 arcmin radius and at almost the same redshift. The unexpected result of this investigation was that the companion cloud contained almost as much mass in $\mathrm{HI}$ as the parent galaxy but was not catalogued in the optical. To answer the question what kind of object the companion is and to determine the kinematics of both objects, follow-up observations were performed: II Zw 33 and its companion (hereafter also called II Zw 33B) were observed with the VLA in its C-array configuration to improve the $u v$-coverage and sensitivity. In addition, the objects were observed through a $B$-band filter with the 4-m telescope of the Kitt Peak National Observatory $^{3}$ to get information about their stellar components.

Dwarf galaxies, $\mathrm{dE}, \mathrm{dSph}$ as well as dIrr, seem to be dominated by Dark Matter. They, as well as late-type spirals, are very well suited for dark matter studies since the absence of a bulge reduces the number of free parameters and thus uncertainties in fitting mass-models. Great progress is being made, using multi-fiber spectrometers, on the velocity distribution in dEs (e.g., Armandroff et al. 1995; for a recent review see Mateo 1994). Moreover, gas rich dIrrs lend themselves to mapping of their velocity fields in $\mathrm{HI}$ to large galactocentric distances since the extent of the gaseous component is larger than the stellar disk. One of the most striking results to emerge from recent studies of dwarf galaxies is that they tend to have much higher dark matter fractions than

${ }^{2}$ The Very Large Array (VLA) is operated by the National Radio Astronomy Observatory (NRAO), a facility of the National Science Foundation, operated under cooperative agreement by Associated Universities, Inc.

${ }^{3}$ The Kitt Peak National Observatory (KPNO), part of the National Optical Astronomy Observatory (NOAO), is operated by the Association of Universities for Research in Astronomy (AURA), Inc., under contract with the National Science Foundation. normal bright galaxies. Related to this is the observation that the dark halo of dwarf irregulars often dominates the dynamics even within the optical radius of the galaxy, which is not the case for more luminous spiral galaxies. (e.g., Carignan 1985; Carignan \& Beaulieu 1989; Jobin \& Carignan 1990; Lake et al. 1990; Côté et al. 1991; Ashman 1992; Brinks \& Taylor 1994).

In this paper, we want to discuss in detail the radio and optical observations (Sec. 2), the H I properties and kinematics (Sec. 3), the mass distribution of the gas and the stars (Sec. 4), and a possible dark matter content via dynamical modelling (Sec. 5). In Sec. 6 we will briefly discuss the possibility to determine the dark matter content of the system by assuming it to be bound. A summary of the results is given in Sec. 7. In the course of this work, an improved scheme was conceived to derive predicted rotation curves based on the observed mass distribution. This is discussed in the Appendix.

\section{OBSERVATIONS \\ 2.1 Radio Observations}

II $\mathrm{Zw} 33$ and its companion were observed with the VLA on 1986 January 9 (B-array) and 1990 December 29 (Carray) in the $21 \mathrm{~cm}$ line of neutral hydrogen ( $\mathrm{H} \mathrm{I}$ ). In B-array (with baselines between 1 and 54 kilo-wavelengths or 0.21$11.4 \mathrm{~km}$ ) the objects were observed for 225 minutes; in C-array (with baselines between 0.34 and 16 kilowavelengths or $0.073-3.4 \mathrm{~km}$ ) 114 minutes were spent on source. The absolute flux calibration was determined by observing 3C 48 for approximately 10 minutes in each configuration, assuming a flux density of $16.17 \mathrm{Jy}$ according to the Baars et al. (1977) scale. This calibrator was also used to derive the complex bandpass corrections. A nearby calibrator $(0500+019)$ was used as secondary amplitude and phase calibrators and its flux was determined to be $2.26 \mathrm{Jy}$. The $\mathrm{C}$-array data were obtained to achieve a relatively high surface brightness sensitivity whereas the B-array data yield a good angular resolution for an analysis of the kinematics.

The B-array data were taken over a $3.125 \mathrm{MHz}$ bandwidth where we selected the central 32 channels, each having a width of $48.8 \mathrm{kHz}\left(10.5 \mathrm{~km} \mathrm{~s}^{-1}\right)$ after Hanning smoothing. At that time, only one polarization (RR) could be recorded in spectral line mode with the VLA. In C-array the 4IF correlator mode was used. In this mode one IF pair is used to take data over a bandwidth of $1.56 \mathrm{MHz}$ with 64 channels, giving a channel width of $24.4 \mathrm{kHz}\left(5.2 \mathrm{~km} \mathrm{~s}^{-1}\right)$, while the second IF pair is used over a $3.125 \mathrm{MHz}$ bandwidth with 32 channels, each having a width of $97.7 \mathrm{kHz}\left(21.0 \mathrm{~km} \mathrm{~s}^{-1}\right)$, again after Hanning smoothing. Both IFs were recorded with dual polarization (RR and LL). The high-velocity-resolution data were combined with the B-array observations and used for detailed kinematic studies presented in this paper. The B-array observation was centered at $2832.0 \mathrm{~km} \mathrm{~s}^{-1}$ the central velocity of the C-array was shifted accordingly because of the difference in velocity resolution (see Table 1).

The data were edited and calibrated separately for both 
TABLE 1. Setup of the VLA during our observations.

\begin{tabular}{lccc}
\hline \hline VLA configuration & $\mathrm{B}$ & $\mathrm{C}$ & ${\mathrm{B} \& \mathrm{C}^{\mathrm{a}}}$ \\
Date of observation & $1986 \mathrm{Jan} .9$ & $1990 \mathrm{Dec} .29$ & - \\
Time on source & $225 \mathrm{~min}$ & $114 \mathrm{~min}$ & - \\
Total bandwidth & $3.2 \mathrm{MHz}$ & $3.2 / 1.6 \mathrm{MHz}$ & $1.22 \mathrm{MHz}$ \\
No. of channels & 32 & $32 / 63$ & 32 \\
velocity resolution & $10.5 \mathrm{~km} \mathrm{~s}^{-1}$ & $21.0 / 5.2 \mathrm{~km} \mathrm{~s}^{-1}$ & $10.5 \mathrm{~km} \mathrm{~s}^{-1}$ \\
Central velocity & $2832.0 \mathrm{~km} \mathrm{~s}^{-1}$ & $2829.375 \mathrm{~km} \mathrm{~s}^{-1}$ & - \\
Half power beam & $7.8^{\prime \prime} \times 5.3^{\prime \prime}$ & $21.3^{\prime \prime} \times 16.0^{\prime \prime}$ & $8.5^{\prime \prime} \times 6.4^{\prime \prime} \mathrm{d}^{-1}$ \\
Noise per channel & $1.2 \mathrm{mJy} \mathrm{beam}^{-1}$ & $0.76 / 1.48 \mathrm{mJy} \mathrm{beam}^{-1}$ & $1.0 \mathrm{mJy} \mathrm{beam}^{-1}$ \\
\hline \hline
\end{tabular}

${ }^{\mathrm{a}}$ Characteristics of the $\mathrm{B}$ and $\mathrm{C}$ configuration data listed in the first two columns after combination.

${ }^{\mathrm{b}}$ Two bandwidths as a result of using the correlator in mode 4.

c"Natural" weighting.

d " ROBUST $=0.8$ " weighting.

arrays with the AIPS package. ${ }^{4}$ The $u v$ data were inspected and bad data points due to either interference or cross talk between antennae were removed, after which the data were calibrated. We inspected our B- and C-array observations separately; subsequently, we smoothed the $\mathrm{C}$-array velocity resolution to that of our B-array data and combined the $u v$ data to form a single dataset (DBCON) which was used for mapping. This final $u v$ dataset had a bandwidth of $1.22 \mathrm{MHz}$ and a velocity resolution of $48.8 \mathrm{kHz}\left(10.5 \mathrm{~km} \mathrm{~s}^{-1}\right)$. All the results presented in this paper are based on this dataset.

Channels with velocities less than $2748 \mathrm{~km} \mathrm{~s}^{-1}$ and greater than $2916 \mathrm{~km} \mathrm{~s}^{-1}$ were determined to be line-free; these channels determined the continuum emission which was then subtracted from the data in the $u v$ plane (UVLIN). After that, 2 sets of datacubes $(512 \times 512$ pixels $\times 25$ channels) were produced. One was created with MX, using natural weighting, and CLEANed (Högbom 1974; Clark 1980), resulting in a spatial resolution of $10.7^{\prime \prime} \times 8.2^{\prime \prime}$ and an rms noise of $0.8 \mathrm{mJy}$ beam $^{-1}$. The other was obtained using the fairly new task IMAGR. IMAGR uses a ROBUST weighting scheme (Briggs 1995) which combines the high sensitivity of natural weighting with the smooth beam and high resolution of uniform weighting. To get an appropriate ROBUST value for our observation, cubes were produced for different ROBUST values $(-4<$ ROBUST $<4)$ and inspected for beam size and noise. Eventually, we chose a value of ROBUST $=0.8$, resulting in a beamsize of $8.5^{\prime \prime} \times 6.4^{\prime \prime}$ and a rms noise of $1 \mathrm{mJy}$ beam $^{-1}$. We also made a set with higher resolution, using uniform weighting but rejected it because the maps were of too low signal-to-noise. We labeled the two datacubes lowand high-resolution data cube, respectively. Unless otherwise mentioned, we used the high resolution datacube for our analysis. No primary beam correction was performed since II $\mathrm{Zw} 33$ and its companion were both situated within the inner $5^{\prime}$ of the $30^{\prime}$ primary beam.

To separate real emission from the noise, the following procedure was followed: prior to inspection, the natural weighted data cube was convolved to a circular beam with a FWHM of $13^{\prime \prime}$. The smoothed map was then tested at the $2 \sigma$ level; if a pixel fell below this level, the counterpart in the cube was blanked. After that, the remaining peaks were inspected. Emission that was present in 3 consecutive chan-

${ }^{4}$ The Astronomical Image Processing System (AIPS) has been developed by the NRAO. nels was considered to be real whereas all other remaining spikes were considered to be noise and blanked. The final result we called master cube. After that, the master cube was used as a mask to blank the high- and low-resolution data cube. This method assures that the same regions are included when inspecting cubes at different resolutions and with different signal-to-noise ratios.

\subsection{Optical Observations}

II $\mathrm{Zw} 33$ and its companion were observed with the 4-m telescope of the Kitt Peak National Observatory (KPNO) on 1995 March 30. A $2048 \times 2048$ pixel CCD was used leading to a resolution per pixel of $0.47^{\prime \prime}$. The seeing was around $2.5^{\prime \prime}$. The usual calibration steps were followed using the IRAF package. ${ }^{5}$ In total, 7 exposures were made at slightly different positions to get rid of cosmic ray hits and bad pixels. Each exposure was corrected for zero-offset (bias) and was flat-fielded using domeflats. The amount of shift on the CCD between the exposures was determined using the IRAF tasks QPHOT and IMCENTROID. After that, the exposures were combined to create a final image. As it turned out, conditions were not photometric during the observation, therefore no calibration using standard stars was performed. In order to get an approximate calibration for II $\mathrm{Zw} \mathrm{33,} \mathrm{values} \mathrm{were}$ taken from the literature. Unfortunately, values for the apparent blue magnitude of II $\mathrm{Zw} 33$ span a rather wide range. The RC3 catalogue (de Vaucouleurs et al. 1991) quotes a value of $m_{B}=14.1 \mathrm{mag}$ which was most probably taken from the CGCG catalogue ( $m_{\text {phot }}=14.0 \mathrm{mag}$, Zwicky et al. 1961). More recent studies present higher magnitudes, i.e., $m_{B}=14.89 \mathrm{mag}$ (Moles et al. 1987), based on CCD observations or $m_{B}=14.75 \mathrm{mag}$ (Takamiya et al. 1995), using photoelectric photometry from 1964 . We reduced our observation with all values and determined the apparent magnitude per $\operatorname{arcsec}^{2}$ of the sky background and compared this value with the values for a good, moonless night (Unger et al. 1988). Both the RC3 and CGCG catalogue would result in a sky background that is $1.1 \mathrm{mag} \operatorname{arcsec}^{-2}$ brighter than expected whereas the Moles value is far more reasonable $(0.2$ mag $\operatorname{arcsec}^{-2}$ brighter than the background). Our conclusion

${ }^{5}$ IRAF is distributed by National Optical Astronomy Observatories, which is operated by the Association of Universities for Research in Astronomy (AURA), Inc., under contract with the National Science Foundation. 


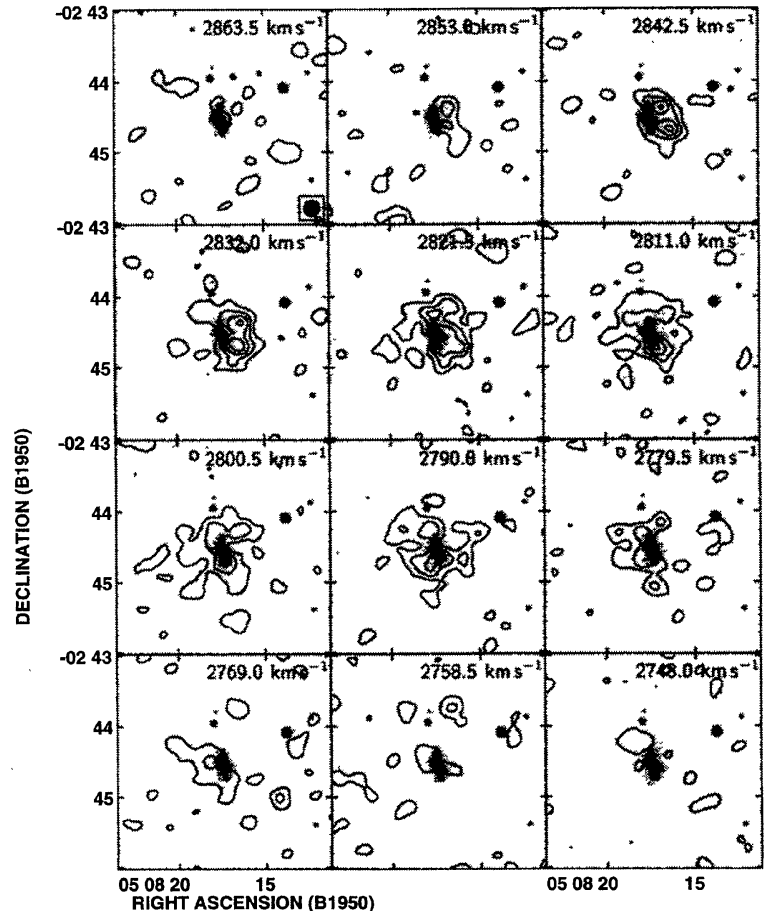

FIG. 1. Mosaic of all channel maps of II Zw 33 in which $\mathrm{H}$ I line emission was detected. Contours are shown at $6,12,18,24 \mathrm{~K}$. The beam size $\left(13^{\prime \prime} \times 13^{\prime \prime}\right)$ is indicated in the top left hand panel. The grey-scale image which is used as background is the optical map ( $B$ band).

therefore is that the values given by the RC3 and CGCG catalogue are incorrect. In the following we therefore used the Moles et al. (1987) value assuming a possible error of the order of $0.1 \mathrm{mag}$.

Coordinates for the maps were obtained using the Mann measuring engine located at the NRAO Array Operation Center in Socorro. The software written for the measuring engine allows the user to establish a coordinate system centered on the region of interest using the Palomar Observatory Sky Survey. By using 14 stars found in our CCD image, it was possible to determine the coordinate system to an accuracy better than one arcsecond, adequate for our needs.

\section{H I STUDIES}

\subsection{Global Properties}

Figures 1 and 2 show a mosaic of all channels in which $\mathrm{H}$ I line emission of II $\mathrm{Zw} 33$ and II $\mathrm{Zw} 33 \mathrm{~B}$ was detected, respectively. To improve the signal-to-noise ratio for extended structure in these maps, the maps were convolved to $13^{\prime \prime} \times 13^{\prime \prime}$. The velocity (redshift) is decreasing in steps of $10.5 \mathrm{~km} \mathrm{~s}^{-1}$ with increasing channel number. The rms noise in those maps is $0.8 \mathrm{mJy}_{\text {beam }}{ }^{-1}$. Notice that the center of emission moves from the right to the left in each map with increasing channel number reflecting the rotation of the objects.

Fig. 3 shows the integrated H I map obtained by the moment analysis of the high-resolution cube of II Zw 33 (north) and its companion (south) as contours. A greyscale image of the optical map is overlaid to show the relative positions of

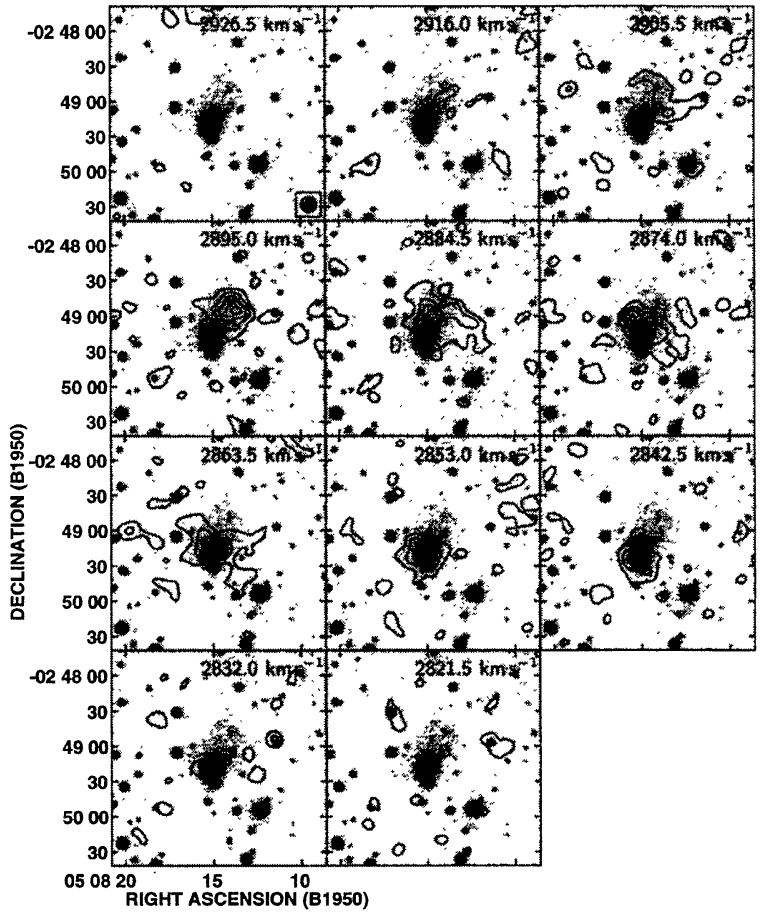

FIG. 2. Mosaic of all channel maps of the companion in which $\mathrm{H}$ I line emission was detected. Contours are shown at $6,12,18,24 \mathrm{~K}$. The beam size $\left(13^{\prime \prime} \times 13^{\prime \prime}\right)$ is indicated in the top left hand panel. The grey-scale image which is used as background is the optical map ( $B$ band).

the objects. Figure 4 contains enlargements of Fig. 3 and show II Zw 33 and its companion, respectively, in more detail ( $\mathrm{H}$ I only). The beamsize is indicated in the bottom right corner.

The global H I profiles of II Zw 33 and II Zw 33B were obtained by summing the total amount of $\mathrm{H} I$ in each map of the high-resolution data cube and are given in Fig. 5. Because, out of practical considerations, we only cleaned down to a level corresponding to about 3 times the rms noise, we made sure, in calculating the integrated fluxes, to correct the values obtained for the contribution coming from the residual, uncleaned flux. We followed in this the prescription given by Jörsäter \& van Moorsel (1995). From the global profiles, midpoint velocities of about $2807 \mathrm{~km} \mathrm{~s}^{-1}$ and 2870 $\mathrm{km} \mathrm{s}^{-1}$ are found for II Zw 33 and II Zw 33B, respectively. Our flux integral for II Zw 33 is $2.98 \mathrm{Jy} \mathrm{km} \mathrm{s}^{-1}$ which translates to an $\mathrm{H}$ I mass of $1.01 \times 10^{9} h_{75}^{-2} \mathrm{M}_{\odot}$ assuming a distance of $37.8 \mathrm{Mpc}\left(h_{75}=1\right.$ and is measured in units of a Hubble constant of $H_{0}=75 \mathrm{~km} \mathrm{~s}^{-1} \mathrm{Mpc}^{-1}$ ). In the case of the companion, the flux integral is $1.74 \mathrm{Jy} \mathrm{km} \mathrm{s}^{-1}$ $\left(0.59 \times 10^{9} h_{75}^{-2} \mathrm{M}_{\odot}\right)$. The $\mathrm{H}_{\mathrm{I}}$ mass of the companion is therefore of the same order of magnitude as the H I mass of the parent galaxy. A comparison of our total $\mathrm{H}$ I profile (both objects) and the profile measured by Thuan \& Martin (1981) is also presented in Fig. 5. Note that there is also a small feature of the companion galaxy in their profile. Since their observation was performed with the NRAO 91-m Green Bank telescope (FWHM $\approx 10^{\prime}$ ) they measured the full flux for II $\mathrm{Zw} 33$ (tracking center). The companion was at about the half power level and this is reflected in the 


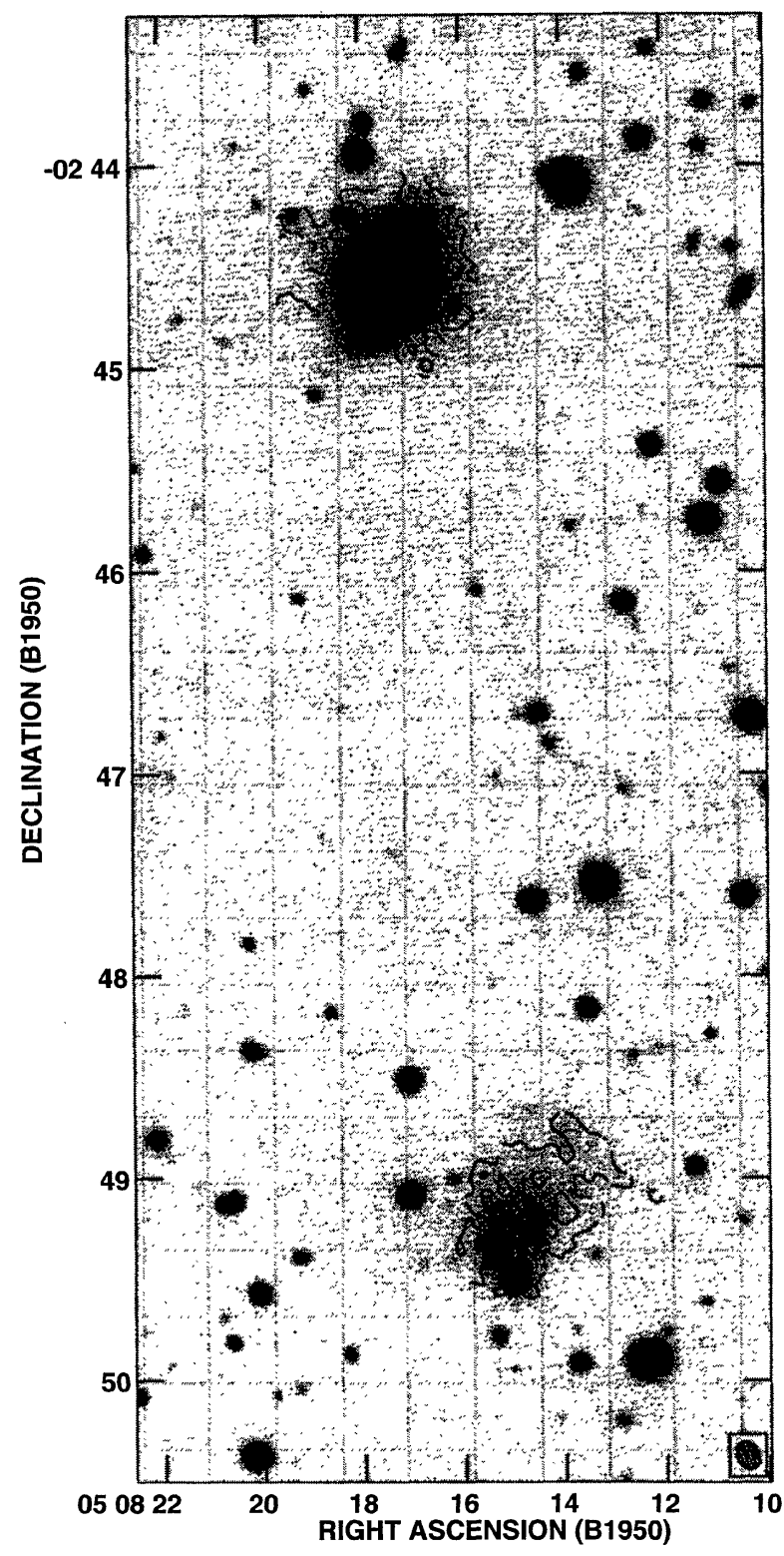

FIG. 3. Integrated $\mathrm{H}_{\mathrm{I}}$ map of II $\mathrm{Zw} 33$ and companion. Contours are shown at $6,18,30,42 \times 10^{20}$ atoms $\mathrm{cm}^{-2}$. The grey-scale image which is used as background is the optical map ( $B$ band).

smaller contribution to the integrated spectrum in the single dish map. A comparison of the two profiles shows that we did not miss any flux due to missing short spacings in our observations.

\subsection{Kinematic Studies}

Figure 6 illustrates the first moment maps (velocity fields) of II Zw 33 and II Zw 33B, respectively. To calculate these maps, the low-resolution data cube was used. Note that the velocity field of II $\mathrm{ZW} 33$ is much more irregular than the one of the companion. This seems related to a higher velocity dispersion of II $\mathrm{Zw} 33$. Figure 7 shows the secondmoment (velocity dispersion) maps of both II Zw 33 and companion. In the central regions of II Zw 33, values are as high as $25 \mathrm{~km} \mathrm{~s}^{-1}$, probably indicating heavy ongoing SF and related SN explosions stirring up the ISM. The companion, on the contrary, shows only moderate values (mean dispersion: $8 \mathrm{~km} \mathrm{~s}^{-1}$ ), leading to the conclusion that not much is going on in terms of SF. This value also agrees with that found in disks of normal spirals (Dickey et al. 1990; Kamphuis 1993).

To derive the rotation curves the task ROTCUR in the GIPSY $^{6}$ package was used (as described by Begeman 1989). This task uses the fact that the projected circular velocity $v_{c, p}$ of an object is related to the real velocity $v_{\mathrm{c}}$, assuming circular orbit of the gas, via:

$$
v_{c, p}(\theta, i, r)=v_{\text {sys }}+v_{\mathrm{c}}(r) \sin (i) \cos \left(\Theta-\Theta_{0}\right),
$$

where $v_{\text {sys }}$ is the systemic velocity, $i$ is the inclination, $\Theta_{0}$ is the position angle of the receding major axis (also referred to as P.A.), and $\Theta-\Theta_{0}$ is the angle measured with respect to the receding major axis of the object. Basically, a set of these parameters that best represents the observed velocity field at all radii must be found by fitting annuli. We proceeded in the following way: First, using best guesses for the systemic velocity $v_{\text {sys }}$ and the kinematic center of the objects, a fit for the inclination $i$, the position angle P.A., and an approximate rotation curve were calculated. Once fits for $i$ and P.A. as a function of radius were found, these values were fixed and $v_{\text {sys }}$ and the center positions were fitted. We repeated this procedure a couple of times until consistent values for the parameters were found. After that, the above mentioned parameters were held at fixed values and a final rotation curve was fitted to the velocity fields.

This procedure worked very well for the companion, although the number of data points for the objects is limited (especially for small radii). The best-fit values are: $i=45^{\circ}$, P.A. $=338^{\circ}, v_{\text {sys }}=2870 \mathrm{~km} \mathrm{~s}^{-1}$. Our fit is consistent with $i$ and P.A. remaining constant as a function of radius. Note that the systemic velocity is in good agreement with the systemic velocity obtained by the global $\mathrm{H}$ I profile. We also checked the results by repeating the procedure with the lowresolution data cube which yielded the same results.

In the case of II Zw 33 this procedure didn't work as well since the velocity field is more disturbed. To reduce the influence of small-scale motions within the ISM in II Zw 33, only the low-resolution data cube was used for further analysis. But even with this data cube we were not able to obtain stable solutions for the inclination. Note that the inclination of an object is usually the least well-constrained parameter, and only the product $v_{c} \sin i$ is well determined. We therefore set the inclination to $i=17^{\circ}$ based on the following considerations: first, the shape of II Zw 33 looks rather circular, suggesting that we are looking at it almost face-on. An inclination of only a few degrees, however, is not possible since we wouldn't see any rotation of the galaxy, and the high velocity dispersion would dominate the total velocity field. The second constraint is then given by the work of Persic \& Salucci (1991) which relates the maximum circular

${ }^{6}$ The Groningen Image Processing System (GIPSY) is distributed by the Kapteyn Astronomical Institute, Groningen, The Netherlands. 

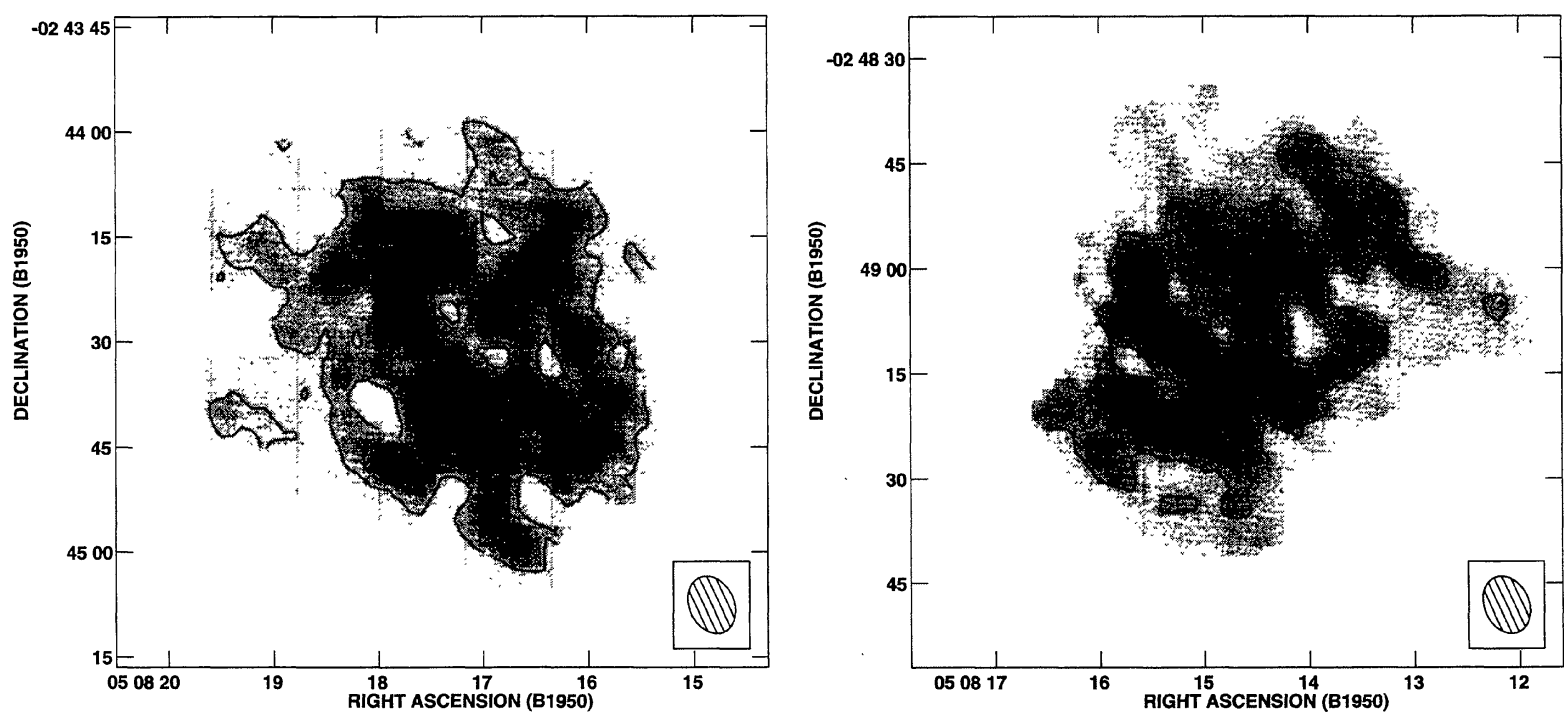

FIG. 4. Integrated $\mathrm{H}$ I map of II $\mathrm{Zw} 33$ (left) and II $\mathrm{Zw} 33 \mathrm{~B}$ (right) obtained with the high-resolution data cube. Contours are shown at $6,18,30,42$ $\times 10^{20}$ atoms $\mathrm{cm}^{-2}$. The greyscale is a linear representation of the $\mathrm{H}$ I surface brightness.

velocity to the absolute blue magnitude of a galaxy. In our case $\left(M_{B}=-18.37 \mathrm{mag}\right.$, see Sec. 4.2$)$ this relationship gives a maximum circular velocity of around $95 \mathrm{~km} \mathrm{~s}^{-1}$ which is in good agreement with an inclination of $17^{\circ}$. We used that value for further analysis, keeping in mind that this value is a major source of uncertainty in our modelling. The final parameters adopted for II Zw 33 are: $i=17^{\circ}$, P.A. $=280^{\circ}$, $v_{\text {sys }}=2807 \mathrm{~km} \mathrm{~s}^{-1}$, and there is no indication for either inclination or position angle to vary with radius (but see also Sec. 5). Again, $v_{\text {sys }}$ is in good agreement with the value obtained by the global $\mathrm{H}$ I profile.

It should be mentioned at this point that this kind of analysis assumes the $\mathrm{HI}$ to be situated in a perfectly thin disk. We know, however, that this is usually not the case for dwarf galaxies (Puche et al. 1992). The uncertainty due to inclination effects can be estimated using the equation:

$$
\cos ^{2} i=\frac{(b / a)^{2}-q^{2}}{1-q^{2}},
$$

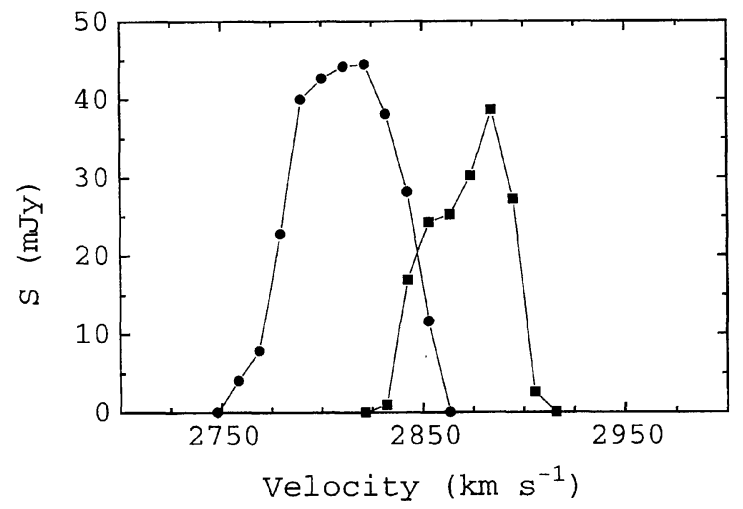

where $q$ is the intrinsic flattening and $a$ and $b$ are the apparent observed major and minor axis. Comparing the values of $i$ for a thin disk $(q=0)$ and a somewhat thicker disk ( $q=0.1$ ) shows that in our cases the correction for $i$ would be $<1 \%$ and could therefore safely be ignored.

\subsection{Rotation Curves}

Using the best-fit values for the systemic velocity, inclination, position angle and center we were able to derive the rotation curves for both galaxies (Fig. 8). The plot shows the rotation curves obtained for the whole galaxy as well as for the receding and approaching halves only, respectively. Note that some values for small radii are missing in the plot. This is due to the fact that there were not enough data points $(<10)$ to get a reliable value. The values for small radii $(<2$ $\mathrm{kpc}$ ) are in reality somewhat higher since we did not perform any correction for beam smearing. We estimated the effect of beam smearing by convolving a theoretical rotation curve

FIG. 5. Left: Global H I profile for II Zw 33 (filled circles) and its companion (filled squares), obtained by integrating the channel maps over the area of the sources after continuum substraction and blanking. Right: Comparison of our global profile (filled squares) with the one measured by Thuan \& Martin (open squares).

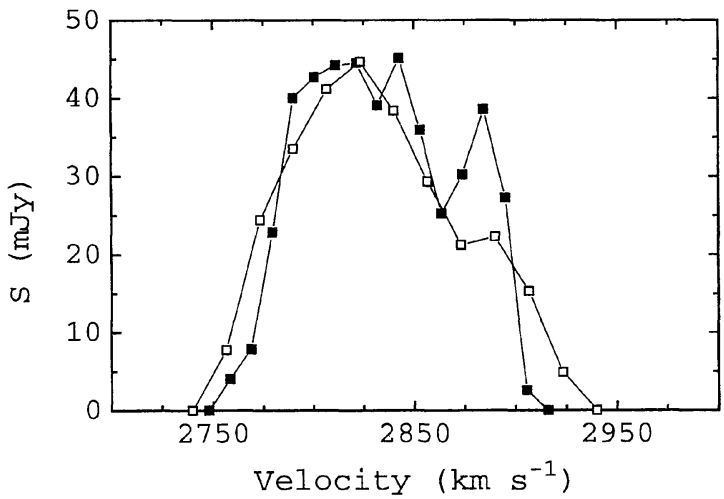



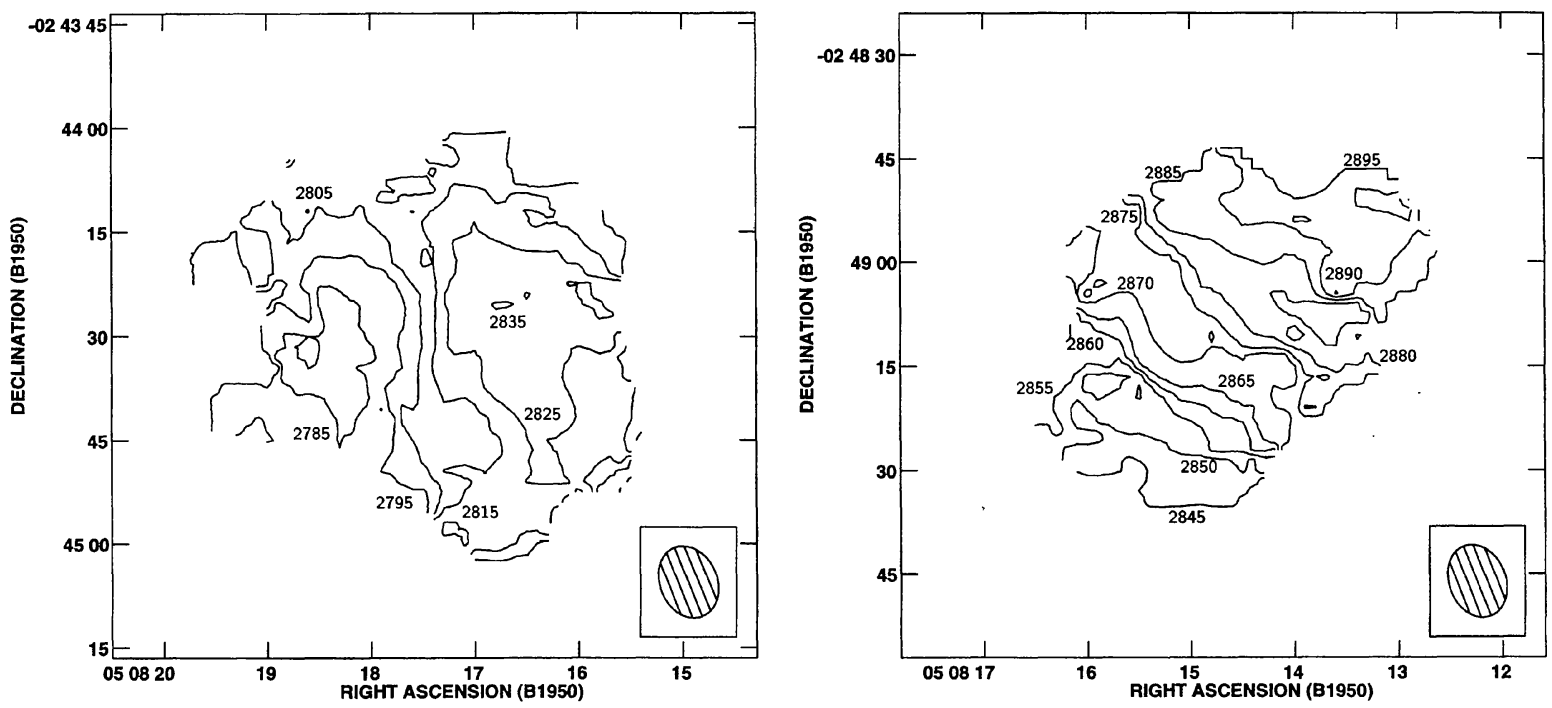

FIG. 6. Isovelocity contours for II $\mathrm{Zw} 33$ (left) and companion (right) obtained from the low-resolution data cube. Velocities are given in $\mathrm{km} \mathrm{s}^{-1}$.

with a model beam of our observation. The result was that the velocities in our observation have been underestimated for small radii $(r<2 \mathrm{kpc})$ by about $5 \%$; we therefore decided not to apply this correction. The adopted errors were obtained by the following procedure: if the difference between the rotation curve for the whole galaxy and that derived for either side was higher than the formal errors given by the least-square analysis for one radius, this value was taken as the error. If not, the value of the least-square error was adopted.

The rotation curve of II Zw 33 is rising until a velocity of $95 \mathrm{~km} \mathrm{~s}^{-1}$ and then decreases to a value of about 50 $\mathrm{km} \mathrm{s}^{-1}$. It has to be mentioned at this point that the decline for $3<\mathrm{r} / \mathrm{kpc}<4.5$ is steeper than Keplerian which is puz- zling. This behavior will be discussed in more detail in Sec. 5. In the case of the companion, the rotation curve increases monotonically with radius up to a value of about $40 \mathrm{~km}$ $\mathrm{s}^{-1}$. It is hard to decide whether the flat part is reached or not. Note that the prominent feature found in the rotation curve of II Zw 33 for small radii is not seen in the companion.

To check the accuracy of our derived parameters, a model velocity field was calculated from the adopted rotation curve and the kinematical parameters using the task VELFI in GIPSY. Given the fact that such models do not include velocity dispersion, a comparison with the measured velocity field gave satisfactory agreement.
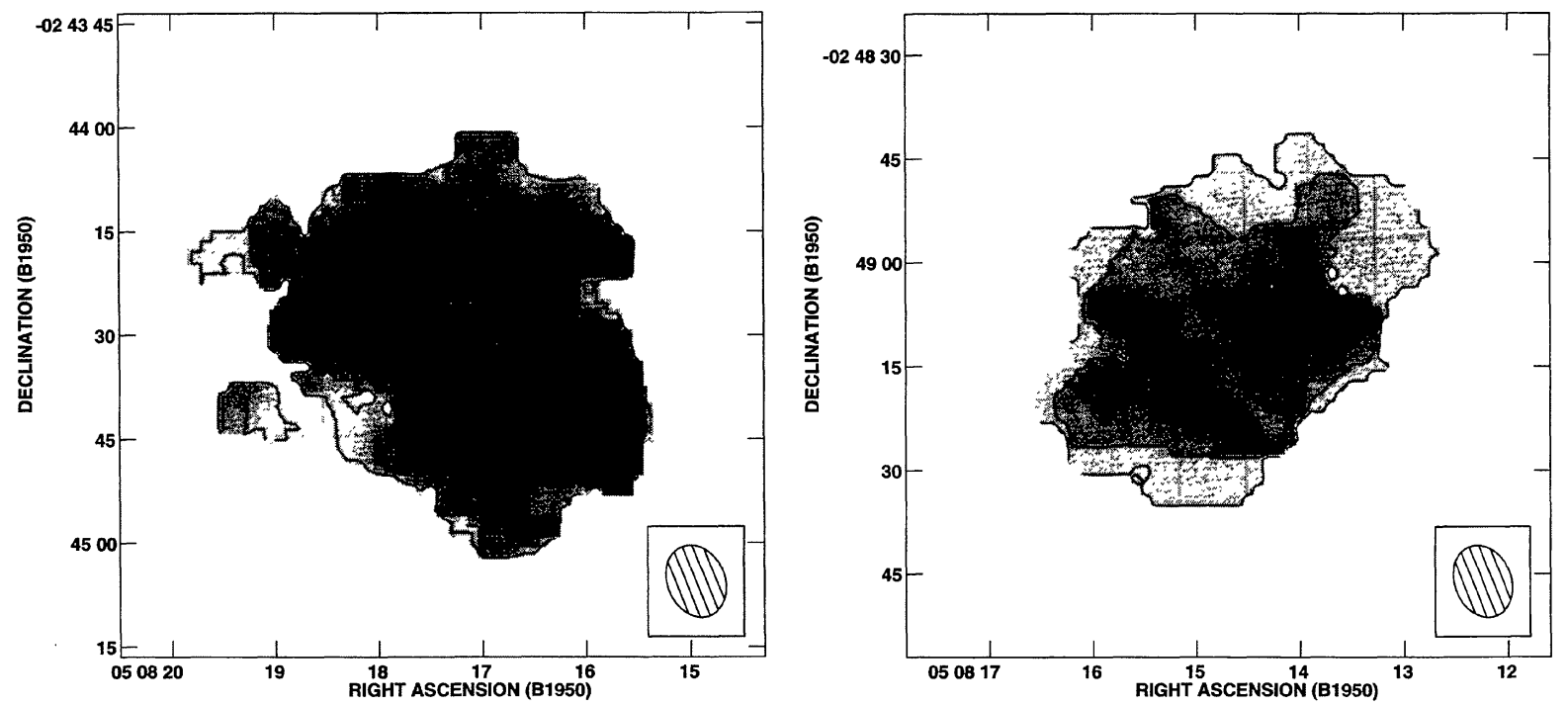

FIG. 7. Velocity dispersion (second moment) maps for II Zw 33 (left) and companion (right) obtained from the low-resolution data cube. Contours are shown at $5,10,15,20 \mathrm{~km} \mathrm{~s}^{-1}$ for $\Pi \mathrm{Zw} 33$ and $3,6,9,12 \mathrm{~km} \mathrm{~s}^{-1}$ for the companion. 

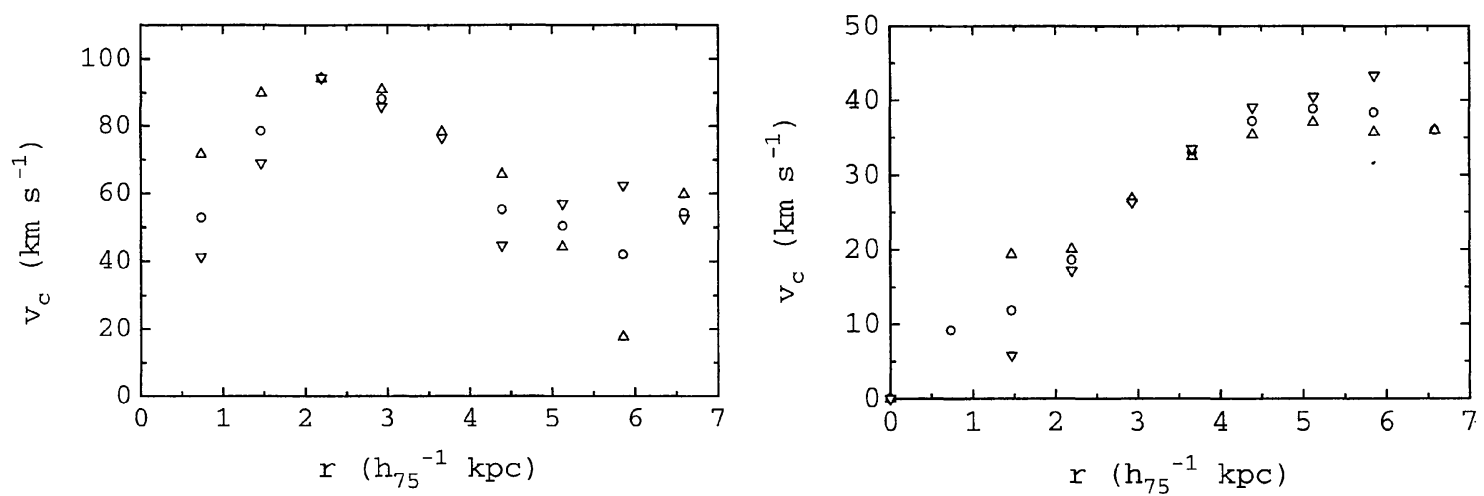

FIG. 8. Rotation curves for II Zw 33 (left) and companion (right) for approaching (down triangle), receding (up triangle) and both halves (circles).

\section{MASS DISTRIBUTIONS}

\subsection{Gaseous Mass}

In order to get the distribution of $\mathrm{H} \mathrm{I}$ mass in II $\mathrm{Zw} 33$ and its companion, the projected surface densities of hydrogen were calculated as a function of radius. This was done by subjecting the integrated $\mathrm{H} \mathrm{I}$ map (high-resolution data) to the task IRING in AIPS. In Fig. 9, the inclination-corrected projected (face-on) $\mathrm{HI}$ density $\sigma$ is plotted as $\mathrm{M}_{\odot} \mathrm{pc}^{-2}$ versus the distance $r$ from the center in kpc for II Zw 33 and II Zw 33B, respectively. A Gaussian function fits the observed $\mathrm{HI}$ density profile well. But since our dynamical analysis in Sec. 5 is based on a modified Hubble density profile for the mass distribution of the hydrogen, we made a fit to the surface density profile with that function as well. It turned out that, although the $\chi^{2}$ value for a Gaussian fit is lower, a modified Hubble profile gives almost the same $\mathrm{H} \mathrm{I}$ mass distribution (and hence potential) for our objects. In addition, the scale lengths of both fits are almost identical. Based on these results we felt confident to fit a modified Hubble profile of the form:

$$
\sigma(r)=\frac{\sigma_{0}}{1+\left(\frac{r}{a_{0}}\right)^{2}}
$$

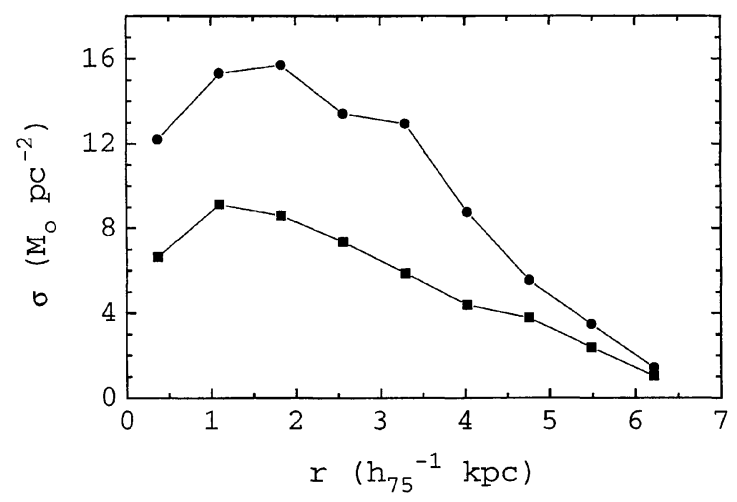

FIG. 9. H I surface density of II Zw 33 (circles) and companion (squares), deprojected in order to represent face-on values. to our data points. The fitting results for both objects are given in Table 2 .

With the help of this fit, the total mass of $\mathrm{HI}$ inside radius $r$ of II Zw 33 and its companion can be calculated by integrating the density profile in rings. For the last measured point $(r=6.6 \mathrm{kpc})$ this gives a total $\mathrm{HI}$ mass of $M_{\mathrm{HI}}=1.06 \times 10^{9} M_{\odot}$ for II $\mathrm{Zw} 33$ and $M_{\mathrm{HI}}=0.58 \times 10^{9} \mathrm{M}_{\odot}$ for II $\mathrm{Zw} 33 \mathrm{~B}$. These values are in good agreement with the ones obtained from the global $\mathrm{H} \mathrm{I}$ profile. In order to get values for the total gaseous mass, one has to know the abundances of the other elements in our objects. To correct for the contribution of $\mathrm{He}$, we adopted a correction of the hydrogen mass by 1.35 . The problem we face is that the abundances of ionized and molecular hydrogen are hard to estimate. $\mathrm{H} \alpha$ images (Brinks 1990) suggest that ionized hydrogen is present in II Zw 33 but can hardly be detected in the companion. Since no $\mathrm{CO}$ observations are available it is almost impossible to give any estimates of the $\mathrm{H}_{2}$ content. Recent CO observations (Israel et al. 1995) suggest that in dwarf galaxies the $\mathrm{H}_{2}$ to $\mathrm{H}$ I ratio is much lower than that for latetype spirals, i.e., $M_{\mathrm{H}_{2}} / M_{\mathrm{H}} \leqslant 0.03$ compared to $M_{\mathrm{H}_{2}} / M_{\mathrm{HI}}=0.4$ for Sc spirals or $M_{\mathrm{H}_{2}} / M_{\mathrm{HI}}=0.1$ for Scd, Sd, and $\mathrm{Sm}$ galaxies (Young \& Knezek 1989). One cannot exclude, though, that in metal-poor dwarf galaxies CO is underabundant whereas $\mathrm{H}_{2}$ abundances may be normal. Therefore a non-detection of $\mathrm{CO}$ emission may not exclude a large abundance of molecular hydrogen. Furthermore, significant amounts of $\mathrm{CO}$ might exist but at very low excitation temperatures, rendering any detection difficult. This would mean that the $\mathrm{CO}$ to $\mathrm{H}_{2}$ conversion factor would be much higher than for spiral galaxies (Sage et al. 1992; Israel et al. 1995). We therefore adopted, somewhat arbitrarily, an intermediate

TABLE 2. Fitting results of $\mathrm{H} I$ analysis.

\begin{tabular}{lcc}
\hline & II Zw 33 & II Zw 33B \\
\hline Systemic velocity $v_{\text {sys }}$ & $2807 \mathrm{~km} \mathrm{~s}^{-1}$ & $2870 \mathrm{~km} \mathrm{~s}^{-1}$ \\
Inclination $i$ & $17^{\circ}$ & $45^{\circ}$ \\
Position angle P.A. & $280^{\circ}$ & $338^{\circ}$ \\
Central density $\sigma_{0}$ & $15.89 \mathrm{M}_{\odot} \mathrm{pc}^{-2}$ & $8.82 \mathrm{M}_{\odot} \mathrm{pc}^{-2}$ \\
Scale length $a_{0}$ & $4.15 \mathrm{kpc}$ & $4.09 \mathrm{kpc}$ \\
H I mass $M_{\mathrm{HI}}$ & $1.01 \times 10^{9} \mathrm{M}_{\odot}$ & $0.59 \times 10^{9} \mathrm{M}_{\odot}$ \\
\hline \hline
\end{tabular}



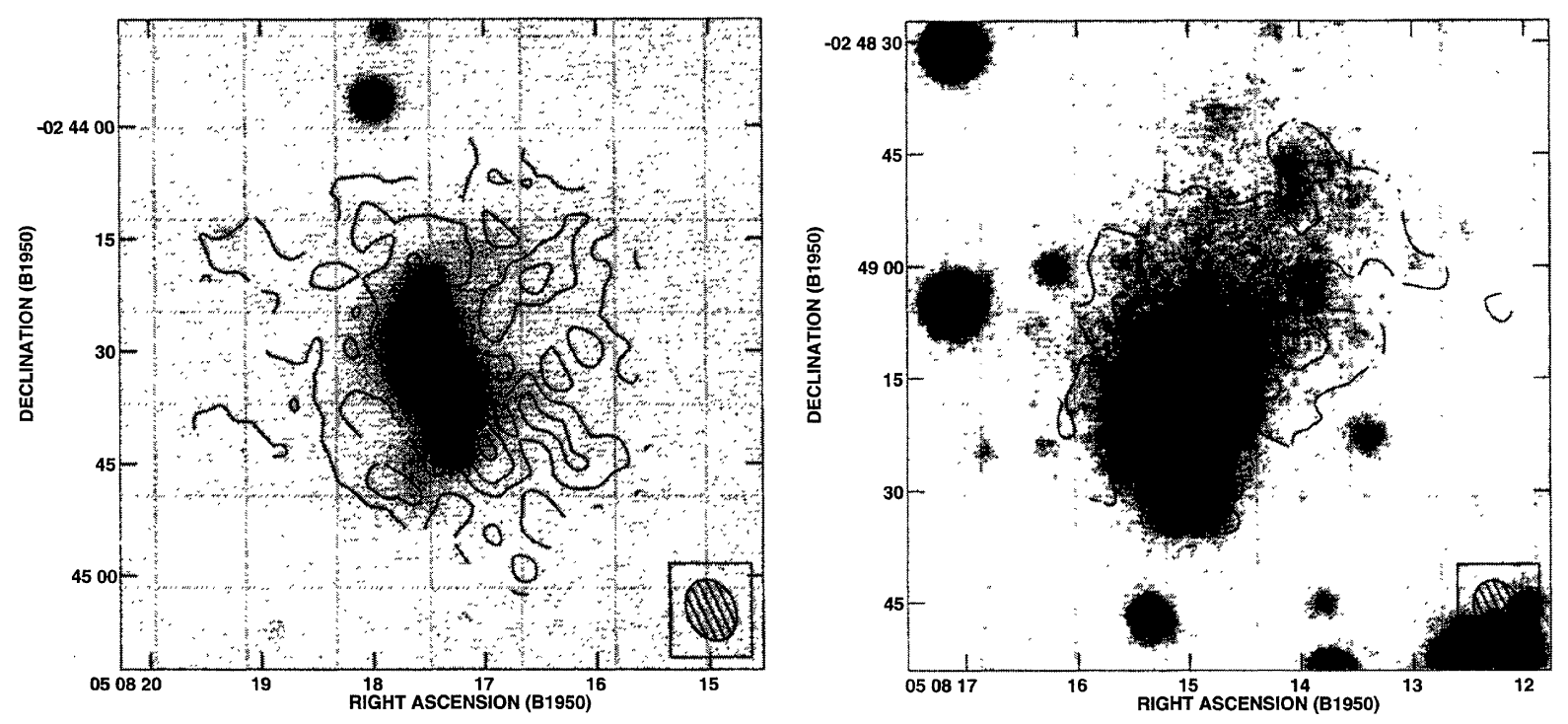

FIG. 10. B band images of II ZW 33 (left) and companion (right). The overlaid contours represent the integrated H I map.

ratio of $M_{\mathrm{H}_{2}} / M_{\mathrm{I}}=0.1$, keeping in mind that this value is a major uncertainty, with a possible error of $\pm 100 \%$. To correct for $\mathrm{He}$ and $\mathrm{H}_{2}$ we therefore multiplied the amount of $M_{\mathrm{H}}$ by a factor of 1.48 . Doing that, we assumed that the $\mathrm{H}$ I distribution is a tracer for $\mathrm{He}$ and $\mathrm{H}_{2}$. We also corrected for the amount of ionized hydrogen ( $\mathrm{H} \mathrm{II}$ ) by taking the average value of $M_{\mathrm{H} \mathrm{II}} / M_{*} \approx 0.15$ (with an assumed error of \pm 0.1 ) for blue compact dwarf galaxies (Campos-Aguilar et al. 1993). Note that in this case the blue stars are used as a tracer for the ionized hydrogen. Because of the virtual absence of ionized gas in the companion, no such correction was performed for II $\mathrm{Zw} 33 \mathrm{~B}$.

\subsection{Stellar Mass}

Figure 10 shows II $\mathrm{Zw} 33$ and its companion in the $B$ band. The overlaid contours represent the integrated H I map. Note that we chose the grey-scale range to be quite different for both objects in order to be able to show the faint emission of the companion. Also note that the $\mathrm{H}$ I emission in II $\mathrm{ZW}$ 33 is more extended than the optical counterpart, offering the possibility to map the rotation curve out to radii larger than the optical radius. The companion is a previously optically undetected object and shows only very weak optical emission. The bright spot to the south was determined to be a foreground star by fitting Gaussians to the brightness profile; moreover, it subtracts out when creating $\mathrm{H} \alpha$ images from on- and off-band CCD frames. There is very weak extended emission within the entire $\mathrm{H}$ I envelope although most of it comes from the southern part. In this area, some low-level SF is going on, as indicated by faint wisps of $\mathrm{H} \alpha$ emission (Brinks 1990). With all that information, II Zw 33B can probably best be classified as a low surface brightness galaxy.

The apparent B magnitude of II Zw 33 is $m_{B}=14.89 \mathrm{mag}$ (Moles et al. 1987) which translates to an absolute magnitude of $M_{B}=-18.37 \mathrm{mag}$, assuming a Hubble constant of
$H_{0}=75 \mathrm{~km} \mathrm{~s}^{-1} \mathrm{Mpc}^{-1}$ (this value is corrected for an assumed galactic extinction in the blue band of $A_{B}=0.37$ (RC3)). II Zw 33 therefore lies at the upper brightness limit for dwarf galaxies (usually taken as $M_{B}=-18 \mathrm{mag}$ ) or at the lower brightness limit for late-type spiral galaxies. Using the absolute magnitude we can estimate the total blue luminosity via:

$$
L_{B}=10^{-0.4\left(M_{B}-\mathrm{M}_{\mathrm{B} \odot}\right)} \mathrm{L}_{\mathrm{B} \odot}=3.31 \times 10^{9} \mathrm{~L}_{\mathrm{B} \odot}
$$

with $\mathrm{M}_{\mathrm{B} \odot}=5.43 \mathrm{mag}$. The ratio of the $\mathrm{H}$ I mass to blue luminosity yields for II $\mathrm{Zw} 33: M_{\mathrm{H}_{\mathrm{I}}} / L_{B}=0.31\left(\mathrm{M}_{\odot} / \mathrm{L}_{\mathrm{B} \odot}\right)$.

The apparent blue magnitude of the companion was determined to be $m_{B}=17.44 \mathrm{mag}$, which corresponds to an absolute blue magnitude of $M_{B}=-15.82 \mathrm{mag}$. This translates into a total blue luminosity of $L_{B}=0.32 \times 10^{9} L_{B} \odot$. The stellar blue luminosity of the companion is therefore an order of magnitude lower than in the parent galaxy. The ratio of the $\mathrm{HI}$ mass to blue luminosity for the companion is: $M_{\mathrm{H}_{\mathrm{I}}} / L_{B}=1.83\left(\mathrm{M}_{\odot} / \mathrm{L}_{\mathrm{B} \odot}\right)$.

Figure 11 shows the B luminosity profile of II $\mathrm{Zw} \mathrm{33,}$ corrected to face-on. This profile was obtained again using the task IRING in AIPS. It is important to state that the best-fit values for the center, inclination and position angle of the $\mathrm{H}$ I data were assumed to be also valid for the optical galaxy in this calculation. We proceeded that way although we are well aware of the optical shape of II $\mathrm{Zw} 33$ and that we average out the prominent star-forming bar. Because we assumed that the kinematics of the gas can be used to determine the orientation of the galaxy, the B luminosity shows some flattening for small radii. As we know from other studies (Taylor et al. 1995, 1996) the SF taking place in H II galaxies in general does not necessarily coincide with the dynamical center. The profile is almost purely exponential for radii $r>1 \mathrm{kpc}$. The best fit yields an extrapolated central surface brightness of 20.4 mag $\operatorname{arcsec}^{-2}$ and a scale length of $0.96 \mathrm{kpc}$. Using this fit the total blue luminosity inside radius $r$ was calculated and used for our dynamical modelling. Note 


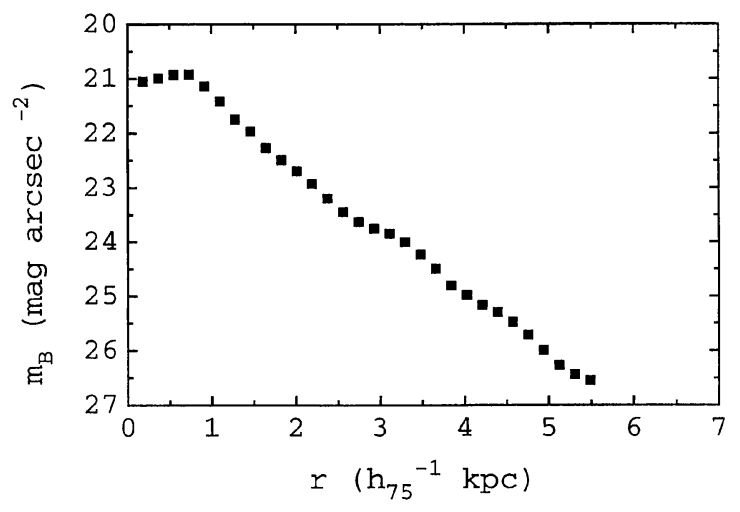

FIG. 11. B luminosity profile of II $\mathrm{Zw} 33$ deprojected to a face-on orientation.

that this luminosity has to be considered as a lower limit since no correction for internal absorption was applied.

We did not perform a similar analysis in the case of II $\mathrm{Zw}$ 33B since, obviously, the stellar mass is not uniformly distributed but clumpy to the south. The total blue luminosity of the stars obtained by the absolute magnitude of II Zw 33B was therefore used.

The optical properties of II Zw 33 and its companion are summarized in Table 3.

\section{DYNAMICAL MODELLING}

To calculate the expected rotation curves for both objects we used an improved method which is described in Appendix A. The basic idea of the approach presented there is to develop a simplified method to obtain rotation curves for flattened mass-distributions. As shown in the Appendix, the circular velocity $v_{\mathrm{c}}$ of a test particle in the $z=0$ plane of a flattened mass distribution can be calculated via:

$$
v(r)=\Gamma(r) \sqrt{G \frac{M(r)}{r}},
$$

where $M(r)$ is the total observed mass inside radius $r$. In the Appendix, we derive the correction function $\Gamma(r)$ which ac-

TABLE 3. Optical properties of II Zw 33 and its companion.

\begin{tabular}{|c|c|c|}
\hline & II Zw 33 & II Zw 33B \\
\hline Right ascension (1950.0) & $05^{\mathrm{h}} 08^{\mathrm{m}} 17^{\mathrm{s}} .39$ & $05^{\mathrm{h}} 08^{\mathrm{m}} 14^{\mathrm{s}} .69$ \\
\hline Declination (1950.0) & $-02^{\circ} 44^{\prime} 29^{\prime \prime} 2$ & $-02^{\circ} 49^{\prime} 10^{\prime \prime} 4$ \\
\hline Apparent magnitude (B) & $14.89 \mathrm{mag}^{\mathrm{a}}$ & $17.47 \mathrm{mag}$ \\
\hline Adopted distance ${ }^{\mathrm{b}}$ & $37.8 h_{75}^{-1} \mathrm{Mpc}$ & $37.8 h_{75}^{-1} \mathrm{Mpc}$ \\
\hline Scale: $1^{\prime \prime}$ corresponds to & $183 h_{75}^{-1} \mathrm{pc}$ & $183 h_{75}^{-1} \mathrm{pc}$ \\
\hline Galactic extinction $(B)^{c}$ & $0.37 \mathrm{mag}$ & $0.37 \mathrm{mag}$ \\
\hline Corrected distance modulus & $\mathrm{m}_{\mathrm{B}}-\mathrm{M}_{\mathrm{B}}=33.2$ & $\mathrm{~m}_{\mathrm{B}}-\mathrm{M}_{\mathrm{B}}=33.2$ \\
\hline Absolute magnitude $(\mathrm{B})^{\mathrm{b}}$ & $-18.37 \mathrm{mag}$ & $-15.82 \mathrm{mag}$ \\
\hline$L_{B}$ & $3.3 \times 10^{9} \quad \mathrm{~L}_{\mathrm{B} \odot}$ & $3.2 \times 10^{8} \quad L_{\mathrm{B} \odot}$ \\
\hline$M_{\mathrm{H}_{\mathrm{I}}} / L_{B}$ & $0.31 \mathrm{M}_{\odot} / \mathrm{L}_{\mathrm{B} \odot}$ & $1.83 \mathrm{M}_{\odot} / \mathrm{L}_{\mathrm{B} \odot}$ \\
\hline Central surface brightness & $20.4 \mathrm{mag} \operatorname{arcsec}^{-2}$ & - \\
\hline Scale length & $0.96 \mathrm{kpc}$ & - \\
\hline
\end{tabular}

Taken from Moles et al. (1987).

${ }^{\mathrm{b}} \mathrm{H}_{0}=75 \mathrm{~km} \mathrm{~s}^{-1} \mathrm{Mpc}^{-1}$.

${ }^{\mathrm{c}}$ Taken from the RC3.

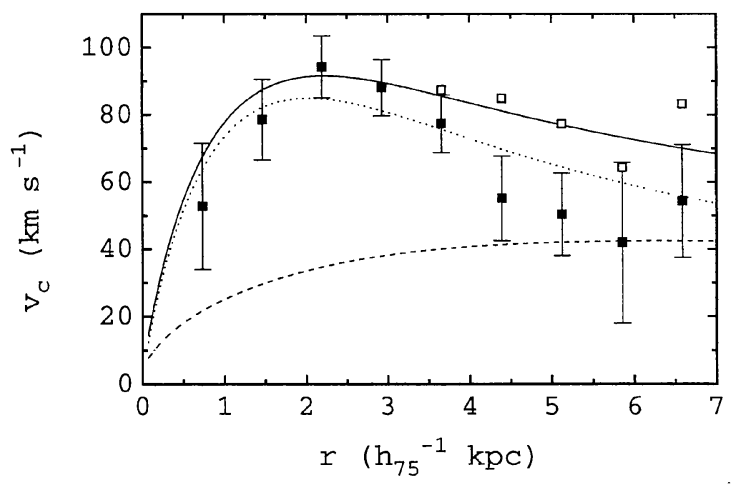

FIG. 12. Expected rotation curves calculated for II Zw 33 based on the gas-component only (dashed), the stellar component only (dotted), and the total observed mass (full line). The filled squares represent the fitted rotation curve with appropriate error bars. The open squares represent the fitted rotation curve when allowing for a possible change in inclination from $17^{\circ}$ to $11^{\circ}$ for large radii.

counts for the fact that in galaxies we are in general not dealing with spherical but rather with flattened mass distributions. As discussed there, this correction function depends on the shape and the density profile of a given mass distribution. Using the results of Appendix A we are able to calculate the expected rotation curves for both the stellar and gaseous component of II Zw 33 and its companion. Doing that, we assumed the gaseous component to be distributed in a flattened ellipsoid ( $q=0.1$ ) which is a good approximation for dwarf galaxies (Puche et al. 1992) and used our fitted modified Hubble profile (Sec 4.1). The stellar component was fitted with a thin exponential disk (Sec. 4.2).

Figure 12 shows the rotation curves expected for the gas component ( $\mathrm{H}$ I corrected for $\mathrm{He}$ and $\mathrm{H}_{2}$ ), the stellar component (assuming $M_{*} / L_{B}=1.0\left(\mathrm{M}_{\odot} / \mathrm{L}_{\mathrm{B} \odot}\right)$ and corrected for the $\mathrm{H}$ II content) and the total mass (stars and gas combined) for II $\mathrm{Zw}$ 33. The points represent the observed rotation curve with appropriate error bars (as discussed in Sec. 3.3). The mass to blue-light ratio of the stars was chosen exploring several $M_{*} / L_{B}$ values in the range of $0.5<M_{*} / L_{B}\left(\mathrm{M}_{\odot} / \mathrm{L}_{\mathrm{B} \odot}\right)<1.5$. A value of $M_{*} / L_{B}=1.0$ fits the steep rise for small radii best and was therefore adopted.

One result of this investigation is that no dark matter is needed for radii $r<6.6 \mathrm{kpc}$ in II $\mathrm{Zw} 33$ which is a major difference to other dIrr or late-type galaxies studied thus far (e.g., Carignan 1985; Carignan \& Beaulieu 1989; Jobin \& Carignan 1990; Lake et al. 1990; Côté et al. 1991; Ashman 1992; Brinks \& Taylor 1994). Notice also that, assuming $M_{*} / L_{B}=1.0\left(\mathrm{M}_{\odot} / \mathrm{L}_{\mathrm{B} \odot}\right)$, the prominent feature in the observed rotation curve is beautifully reproduced by the stellar component of II Zw 33. A major problem we face is the fact that the decline for $r>3 \mathrm{kpc}$ is steeper than Keplerian. This is obviously not possible. One explanation might be that the error bars are fairly large for large radii and that, within the error bars, everything is fine. Most probably, however, this apparent decline is due to a change in inclination whereas we set the inclination to a fixed value for all radii (see Sec. 3.2.). 


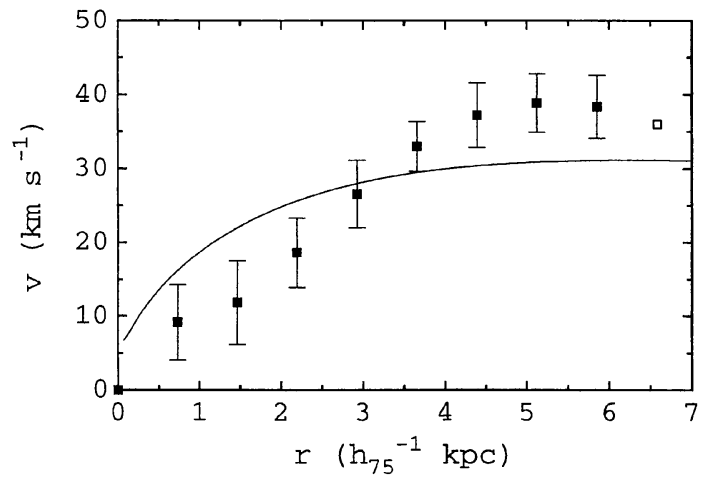

FIG. 13. Expected rotation curve for II $\mathrm{Zw} 33$ based on the gas component only. The filled squares represent the fitted rotation curve with appropriate error bars. For the last measured point (open square) data for only one side is presented.

The plotted open squares show how the shape of the observed rotation curve would change if there would be a small change in inclination from $17^{\circ}$ to $11^{\circ}$ for larger radii. Such a warp could be explained as due to the interaction with the companion. In that case, however the problem would be why we observe a warp only in the more massive galaxy. Another possibility could be that the last measured points of the rotation curves are correct and that there is a change in inclination for smaller radii in such a way that the hump is not that prominent. This then would require $M_{*} / L_{B}$ $=0.5\left(\mathrm{M}_{\odot} / \mathrm{L}_{\mathrm{B} \odot}\right)$ but in that case the peak velocity would fall well below the value quoted by Persic \& Salucci (1991) (Sec. 3.2) and therefore does not seem to be an attractive proposition.

The major sources of uncertainty should be summarized at this point: the inclination was fixed at $17^{\circ}$, with an uncertainty of $\pm 3^{\circ}$. This yields an error in velocity of the observed rotation curve of $\pm 20 \%$. The combined mass of $\mathrm{HI}$, $\mathrm{H}_{2}$, and $\mathrm{He}$ was assumed to be $1.45 \times M_{\mathrm{HI}}$ with an uncertainty of about $10 \%$ (leading to an error of $\pm 5 \%$ in velocity), mainly due to the lack of information on the relative abundance of $\mathrm{H}_{2}$. A major uncertainty is the mass-to-light ratio of the stars. Usually, $M_{*} / L_{B}$ relations for dwarf galaxies span a rather wide range (Thuan \& Martin 1981). A value $M_{*} / L_{B}>1\left(\mathrm{M}_{\odot} / \mathrm{L}_{\mathrm{B} \odot}\right)$ would raise the rotation curve produced by the stellar component by a factor of $\sqrt{M_{*} / L_{B}}$. Higher values for $M_{*} / L_{B}$ would therefore overshoot the observed rotation curve and could only be explained by reducing the inclination of II Z $\mathrm{Zw} 33$.

The same analysis was performed for II $\mathrm{Zw} 33 \mathrm{~B}$. Figure 13 shows the rotation curve expected for the gas component only, and the actually observed one (points with error bars). The stellar component has not been modelled (see Sec. 4.2) but adding the total stellar mass (assuming again $M_{*} / L_{B}$ $\left.=1.0\left(\mathrm{M}_{\odot} / \mathrm{L}_{\mathrm{B} \odot}\right)\right)$ would raise the predicted rotation curve of the gas by about $5 \mathrm{~km} \mathrm{~s}^{-1}$ at the last measured point, which fits the observed rotation curve very well. This means that, also in the companion, no dark matter needs to be invoked to explain the observed rotation curve. Major uncertainties are: the $\mathrm{H}_{2}$ and $\mathrm{HII}$ content, resulting in an error of $13 \%$ in gaseous mass, and $M_{*} / L_{B}$ of the stellar component. Again,
$M_{*} / L_{B}>1.0\left(\mathrm{M}_{\odot} / \mathrm{L}_{\mathrm{B} \odot}\right)$ would overshoot the observed rotation curve. This leads to the interesting conclusion that, based on the kinematical analysis we can set an upper limit to the $M_{*} / L_{B}$ ratio. Our result $\left(M_{*} / L_{B} \simeq 1.0\left(\mathrm{M}_{\odot} / \mathrm{L}_{\mathrm{B} \odot}\right)\right)$ implies that we are dealing with a fairly young stellar population in the companion.

Things look somewhat different if we vary the distance to our object. The adopted distance of $37.8 \mathrm{Mpc}$ (assuming $H_{0}=75 \mathrm{~km} \mathrm{~s}^{-1} \mathrm{Mpc}^{-1}$ ) is based on the naive assumption of a uniformly expanding Universe. We note that II Zw 33 and its companion are situated about half-way between us and the galaxy cluster Abell 539 (Ostriker et al. 1988). Since the observed luminous mass (i.e., $\mathrm{H}$ I and stars) scales with $d^{2}$ but the dynamical mass only with $d$ this means that the ratio of the dynamical mass, $M_{\text {dyn }}$, to the observed total mass, $M_{\text {tot }}$, scales as $d^{-1}$. In other words, if II $\mathrm{Zw} 33$ were situated at $28.4 \mathrm{Mpc}$ (corresponding to $H_{0}=100 \mathrm{~km} \mathrm{~s}^{-1} \mathrm{Mpc}^{-1}$ or $h_{75}=1.33$ ) our total observed mass must be higher by a factor of 1.33 to explain the observed rotation curve. If we don't want to invoke any dark matter component, the only parameter we can vary is the stellar mass to light ratio. In that case, the rotation curve of II $\mathrm{Zw} 33$ could be explained with $M_{*} / L_{B} \approx 1.5\left(\mathrm{M}_{\odot} / \mathrm{L}_{\mathrm{B} \odot}\right)$. In the companion, the mass to blue-light ratio of the stars would have to be as high as 2 . For a larger distance of $56.8 \mathrm{Mpc}$ (corresponding to $H_{0}=50 \mathrm{~km} \mathrm{~s}^{-1} \mathrm{Mpc}^{-1}$ ) we would observe too much mass in both objects. In II Zw 33 this problem could be solved with $M_{*} / L_{B} \approx 0.5\left(\mathrm{M}_{\odot} / \mathrm{L}_{\mathrm{B} \odot}\right)$ whereas we would overshoot the observed rotation curve in the companion even with $M_{*} / L_{B}=0\left(\mathrm{M}_{\odot} / \mathrm{L}_{\mathrm{B} \odot}\right)$ (no stellar component). Therefore, if anything, our adopted distance is likely to be an upper limit.

Although we do not need dark matter to explain our observed rotation curves, the possibility cannot be ruled out that there is some dark matter in both objects, showing its presence only at larger radii (as discussed in Sec. 6). The question remains, however, why our objects are not darkmatter dominated even for smaller radii as is usually found for other dwarf and late-type spirals. In order to resolve that issue, deeper H I observations are needed to probe the kinematics out to larger radii. In addition, broad-band optical observations are necessary to better model the stellar populations in the objects.

\section{II ZW 33 AND II Zw 33B AS A BINARY SYSTEM}

In general, bound pairs offer the attractive possibility of obtaining estimates of dark halo masses at greater galactocentric distances than are usually accessible to $\mathrm{H}$ I rotation curves. In practice, however, such interpretations are not straightforward. Since the geometry of the orbit of our pair is unknown, it is impossible to translate the projected velocity difference $v_{p}$ and separation $r_{p}$ into a real orbital velocity and physical separation. Because of that, statistical methods have usually been applied to a large sample of binary galaxies (Erickson et al. 1987; van Moorsel 1987).

Despite all these problems we couldn't resist to do such an analysis, assuming that II Zw 33 and its companion are a bound system and that we are not observing a chance super- 
position or a chance encounter. In our analysis, we followed the method described by van Moorsel (1987). The total mass $M_{\text {tot }}$ of two point masses describing an orbit around a common center of gravity is given by:

$$
M_{\mathrm{tot}}=\frac{r v^{2}}{G},
$$

where $r$ is the true seperation, $v$ the orbital velocity, and $G$ the gravitational constant. Since the geometry of the pair is not known and only the line-of-sight component of $v, v_{p}$, and the projection of $r$ onto the sky plane, $r_{p}$, can be measured, it is useful to separate the above expression into an observable and a non-observable part, i.e.,

$$
\chi M_{\mathrm{tot}}=\frac{r_{\mathrm{p}} v_{\mathrm{p}}^{2}}{G},
$$

where $\chi$ is the so-called orientation parameter which includes the orientation and the orbital angles (for a more thorough discussion of $\chi$ see van Moorsel (1987)). For circular orbits $(e=0), \chi$ is always $<1$. In addition, doing a Monte Carlo analysis (given a probability distribution of $e$ and assuming random orientations and orbital phases for the orbit) van Moorsel showed that the propability function $P(\chi)$ is sharply peaked for small values of $\chi$. Only for high values of $e, \chi$ may eventually exceed 1 , but $P(\chi)$ is even more strongly peaked towards $\chi=0$. That means that there is a strong tendency to underestimate the total mass.

In our case, with $v_{p}=63 \mathrm{~km} \mathrm{~s}^{-1}$ and $r_{p}=47.8 \mathrm{kpc}$ $\left(4.36^{\prime}\right)$, the total mass is: $M_{\text {tot }}=\chi^{-1} 43.9 \times 10^{9} \mathrm{M}_{\odot}$. Because of the properties of $\chi$, this has to be taken as a lower limit to the total mass of both objects. The sum of our derived masses (II $\mathrm{ZW}$ 33: $5.1 \times 10^{9} \mathrm{M}_{\odot}$, II $\mathrm{Zw} 33 \mathrm{~B}$ : $\left.1.2 \times 10^{9} \mathrm{M}_{\odot}\right)$ is $M_{\text {tot }}=6.3 \times 10^{9} \mathrm{M}_{\odot}$. This mass is therefore an order of magnitude too low to bind the objects, indicating that there likely is a large amount of dark matter surrounding the objects.

The geometry of the pair suggests that the relative orbits are more or less within the plane of the sky. Let us therefore assume that the projected separation of about $50 \mathrm{kpc}$ is close to the true separation. Taylor et al. $(1995,1996)$ show that in their sample of $\mathrm{H}$ II galaxies the difference in velocity between dwarf galaxy and companion is always less than about $150 \mathrm{~km} \mathrm{~s}^{-1}$. Taking these two values, we derive an elapsed time since perigalacticon of about $3 \times 10^{8}$ years. Since the $\mathrm{H}$ II regions in II Zw 33 cannot be older than about $10^{7}$ years, this implies that the current SF seen in II Zw 33 cannot have been caused directly by the passage of its companion. It requires that after initiating a burst $3 \times 10^{8}$ years ago, the $\mathrm{SF}$ process has continued, most likely as envisaged by the theory of stochastic SF.

If we accept the above, i.e., that we are witnessing more than a chance encounter between II Zw 33 and its companion, then there is a clear need for Dark Matter to keep the galaxies bound. Note, though, that there are no compelling reasons to invoke DM to explain the rotation curves and mass distributions of the individual galaxies. Deeper $\mathrm{H}$ I observations might reveal a connection between both objects such as a bridge or tidal tails which would provide unambiguous proof of the objects undergoing an interaction.

\section{SUMMARY}

We have presented a detailed radio and optical study of the dwarf galaxy II Zw 33 and its low surface brightness companion. The main results are:

(1) The companion is a previously undetected object with an apparent blue magnitude of 17.44 mag. Assuming a distance of $37.8 \mathrm{Mpc}$ the absolute blue magnitude is -15.82 mag and II Zw 33B can be classified as a low surface brightness galaxy. We found some indication that star formation is going on at a low level in the south of the companion. The companion might be a dIrr galaxy in its quiescent state (between SF bursts). Note that the companion would not have been discovered at all if II Zw 33 had not been there. II Zw 33 , on the other hand, shows a very prominent SF bar in the optical and has been classified as an $\mathrm{H} \amalg$ galaxy. This active $\mathrm{SF}$ is also reflected in the velocity dispersion maps as well as in the $\mathrm{H} \alpha$ and radio continuum images that are available to us. The current SF bursts that we are witnessing is probably triggered by the interaction with the companion. The apparent blue magnitude of II $\mathrm{Zw} 33$ is 14.89 mag which translates into an absolute blue magnitude of $-18.37 \mathrm{mag}$. II $\mathrm{Zw}$ 33 is therefore situated at the bright end of the classification scheme of dwarf galaxies.

(2) Although the $\mathrm{H}$ I masses of both objects are comparable (II Zw 33: $M_{\mathrm{HI}}=1.01 \times 10^{9} \mathrm{M}_{\odot}$, II $\mathrm{Zw} 33 \mathrm{~B}: M_{\mathrm{H}}$ $=0.59 \times 10^{9} \mathrm{M}_{\odot}$ ), the blue luminosity differs by an order of

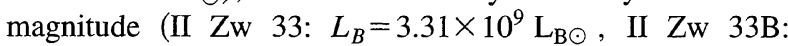
$\left.\mathrm{L}_{\mathrm{B}}=0.32 \times 10^{9} \mathrm{~L}_{\mathrm{B} \odot}\right)$. This results in quite different $\mathrm{H}$ I mass to blue light ratios of $M_{\mathrm{HI}} / L_{B}=0.31\left(\mathrm{M}_{\odot} / \mathrm{L}_{\mathrm{B} \odot}\right)$ for II $\mathrm{Zw} 33$ and $M_{\mathrm{HI}} / L_{B}=1.83\left(\mathrm{M}_{\odot} / \mathrm{L}_{\mathrm{B} \odot}\right)$ for the companion.

(3) Velocity dispersion maps, which show values as high as $24 \mathrm{~km} \mathrm{~s}^{-1}$, confirm that active SF, and its aftermath, have a significant influence on II $\mathrm{Zw} 33$. SF occurs in regions where the $\mathrm{HI}$ surface density exceeds $3 \times 10^{21}$ atoms $\mathrm{cm}^{-2}$. In II $\mathrm{Zw} \mathrm{33B}$, weak SF occurs where the $\mathrm{H}$ I surface density reaches values higher than $2 \times 10^{21}$ atoms $\mathrm{cm}^{-2}$. In the companion we find correspondingly low average values for the velocity dispersion of $8 \mathrm{~km} \mathrm{~s}^{-1}$.

(4) An analysis of the rotation curve of II Zw 33 shows that no dark matter is needed for $r<6.6 \mathrm{kpc}$ (assuming a constant $\left.M_{*} / L_{B}=1\left(\mathrm{M}_{\odot} / \mathrm{L}_{\mathrm{B} \odot}\right)\right)$. This is contrary to what is seen in other dwarf galaxies where the dark matter often dominates the rotation curve even within the optical radius. The analysis of the rotation curve of the companion implies that no dark matter is needed for $r<6.6 \mathrm{kpc}$ either. In the companion, the gaseous component alone is almost entirely able to explain the observed rotation curve. Moreover, we determine an upper limit of $M_{*} / L_{B}=1\left(\mathrm{M}_{\odot} / \mathrm{L}_{\mathrm{B} \odot}\right)$; a higher value would cause the predicted rotation curve to overshoot the observed one. Varying the adopted distance for the system implies that for a larger distance $M_{*} / L_{B}$ needs to be reduced to near zero so as to avoid overshooting the observed curve. Therefore, if anything, the assumed distance of $37.8 \mathrm{Mpc}$ can be considered an upper limit.

(5) There is a striking difference between the two rotation curves of the galaxies: the one for II $\mathrm{Zw} 33$ showing a steep rise to a maximum of $95 \mathrm{~km} \mathrm{~s}^{-1}$ followed by a decline, the one for the companion rising monotonically. This is most 
likely completely due to the fact that II $\mathrm{Zw} 33$ contains a stellar component whereas the companion is dominated by the gas. This is confirmed by the kinematical modeling. A value of $M_{*} / L_{B}=1\left(\mathrm{M}_{\odot} / \mathrm{L}_{\mathrm{B} \odot}\right)$ fits the data best and is valid for both objects.

(6) Assuming $M_{*} / L_{B}=1\left(\mathrm{M}_{\odot} / \mathrm{L}_{\mathrm{B} \odot}\right)$ for both objects and correcting for the masses in $\mathrm{H} \mathrm{II}, \mathrm{H}_{2}$, and $\mathrm{He}$, we get the following total masses for our objects: $M_{\text {tot }}=5.1 \times 10^{9} \mathrm{M}_{\odot}$ (II Zw 33) and $M_{\text {tot }}=1.2 \times 10^{9} \mathrm{M}_{\odot}$ (II Zw 33B). The assumption that the objects are bound (and that we are not observing a chance superposition) suggests that a total mass of at least $44 \times 10^{9} \mathrm{M}_{\odot}$ is needed to bind the system. This means that the amount of observed total mass $\left(6.3 \times 10^{9} \mathrm{M}_{\odot}\right)$ is by far not able to bind the system and that there is need for a huge amount of dark matter surrounding the objects.

Although our data are not sensitive enough to reveal unambiguous proof of an on-going or recent interaction between II Zw 33 and II Zw 33B, there is substantial circumstantial evidence that they do form an interacting, and possibly gravitationally bound, system. Deeper imaging with the VLA could provide further proof of this. The picture which emerges from our study then is that a recent interaction has triggered the SF in the more massive of the two objects. An analysis as performed by Taylor et al. (1994), determining the threshold for the onset of SF in dwarf galaxies, together with deep radio-continuum observations, optical and NIR imaging, and spectroscopy could shed light on why the companion is so different from II $\mathrm{Zw} \mathrm{33,} \mathrm{whereas}$ their $\mathrm{H}$ I distributions and masses are so similar.

F.W. wants to thank the Departamento de Astronomía of Guanajuato for the warm hospitality during his stay in Mexico. F.W. also appreciates the help of the staff of the Array Operation Center, and general support from NRAO during his stay in Socorro, New Mexico. We thank C. Taylor and E. Wilcots for their advice on and help with the optical data reduction and J. Binney for illuminating remarks regarding the Appendix. F.W. also thanks J. Isserstedt who suggested some important clarifications in several conversations. F.W. gratefully acknowledges the "Deutsche Forschungs Gesellschaft (DFG)" for the award of a stipendium in the "Graduiertenkolleg Die Magellanschen Wolken und andere Zwerggalaxien (The Magellanic Clouds and other Dwarf Galaxies)." This research has made use of the NASA/IPAC Extragalactic Database (NED) which is operated by the Jet Propulsion Laboratory, Caltech, under contract with the National Aeronautics and Space Administration and NASA's Astrophysical Data System Abstract Service (ADS).

\section{APPENDIX A: ROTATION CURVE FOR NON-SPHERICAL SYSTEMS}

Calculating the rotation curve for a given flattened mass distribution is rather complicated. Unfortunately, we have to deal with these distributions if we want to study the dynamics of galaxies. If we assume the mass of a galaxy to be spherically distributed, the calculation is fairly easy but we get the wrong result for the rotation curve. However, we get at least a first approximation. Our basic idea was therefore to find correction functions for certain cases that allow us to derive the real rotation curve of a flattened object from theerroneous-spherical case, i.e.,

$$
v(r)=\Gamma(r) \sqrt{G \frac{M(r)}{r},}
$$

where $M(r)$ is the total observed mass inside radius $r$. In order to get the correction function $\Gamma(r)$ one has to derive the rotation curves for non-spherical systems. For an excellent and detailed discussion of this topic, see Binney \& Tremaine (1987). The results presented here are almost all adopted from Binney \& Tremaine.

In general, our problem can be solved by using potential theory which is based on the gravitational potential $\Phi(\vec{x})$ defined by:

$$
\Phi(\vec{x})=-G \int \frac{\rho\left(\vec{x}^{\prime}\right)}{\left|\vec{x}^{\prime}-\vec{x}\right|} d^{3} x^{\prime} .
$$

The force on a unit mass at position $\vec{x}$ is therefore:

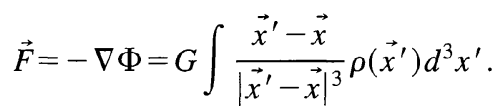

For circular orbits, the condition must hold that the gravitational force (pointing in the radial direction towards the center) equals the centrifugal force, i.e., for a unit mass:

$$
v_{c}^{2}=r|F|=-r \nabla \Phi=-r \frac{d \Phi}{d r} .
$$

With the help of this equation we can in principle calculate the rotation curve $v_{c}(r)$ once we know the potential $\Phi$.

For an ellipsoidal mass-distribution (flattened sphere) the circular velocity at a point with cylindrical coordinates $R$ and $z=0$ (galactic plane) is given by:

$$
v_{c}^{2}(r)=-r \nabla \Phi=4 \pi G \sqrt{1-e^{2}} \int_{0}^{r} \frac{\rho\left(m^{2}\right) m^{2} d m}{\sqrt{r^{2}-m^{2} e^{2}}},
$$

where $e$ is the excentricity of the ellipsoid and $\rho$ is the density of the ellipsoid shell labeled $m$.

Often the density in a galaxy can be fitted fairly well with:

$$
\rho\left(m^{2}\right)=\rho_{0}\left[1+\left(\frac{m}{a_{0}}\right)^{2}\right]^{-(3 / 2)},
$$

where $a_{0}$ is the core radius and $\rho_{0}$ is the central density of the mass distribution. In the limit $e \rightarrow 0$ this reduces to $j(r)=j_{0}\left(1+\left(r / a_{0}\right)^{2}\right)^{-3 / 2}$ which, integrated along the line of sight gives a projected surface density of $\sigma(r)$ $=2 j_{0} a_{0}\left(1+\left(r / a_{0}\right)^{2}\right)^{-1}$ - the so-called modified Hubble profile. Inserting this density into the above equation yields:

$$
v_{c}^{2}(r)=4 \pi G \rho_{0} a_{0}^{3} \frac{\sqrt{1-e^{2}}}{r} k[\mathrm{~F}(\Theta, k)-\mathrm{E}(\Theta, k)],
$$

where 


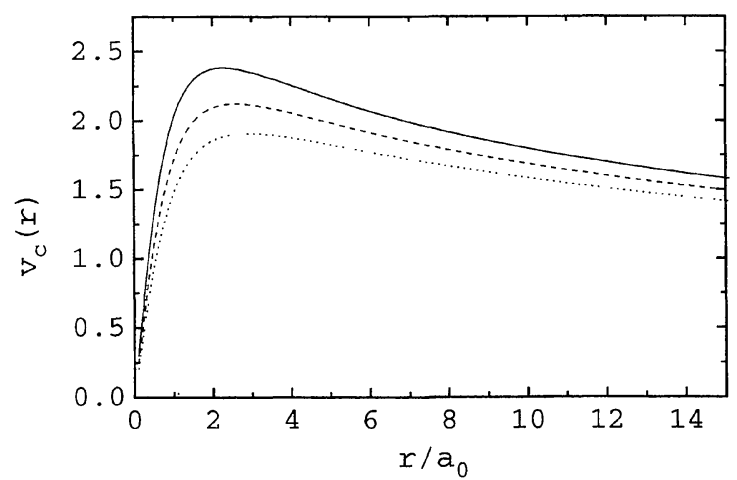

FIG. 14. Circular speed versus radius for three bodies with the same face-on projected density profile (the modified Hubble profile) but different axis ratio $q=b / a$. Curves are plotted for $q=1$ (dotted, spherical case), $q=0.5$ (dashed) and $q=0.1$ (full curve), respectively (adapted from Binney \& Tremaine).

$$
k=\left[\left(\frac{a_{0} e}{r}\right)^{2}+1\right]^{-(1 / 2)}, \quad \Theta=\arcsin \sqrt{\frac{e^{2} a_{0}^{2}+r^{2}}{a_{0}^{2}+r^{2}}},
$$

Note that $\mathrm{F}(\Theta, k)$ and $\mathrm{E}(\Theta, k)$ are incomplete elliptic integrals.

Figure 14 shows the circular velocity plotted versus radius for three bodies with the same face-on projected density profile (the modified Hubble profile) but different axis ratio $q=b / a$. Note that although all three bodies have the same total mass inside a spheroid of given major axis, $v_{c}$ increases with flattening $1-q$. This means that if we know the projected density profile of a galaxy, but assume that the galaxy has a spherical mass-distribution, we will get velocities that are too low. However, knowing this theoretical result we can now correct the spherical result by multiplying it with a correction function $\Gamma(r)$. This correction function is simply the ratio of the rotation curve of the flattened mass distribution to the one of the spherical counterpart and is plotted in Fig. 15.

A function that fits this correction function well is: $\Gamma(r)=a(r+b)^{-c}+d$ and a best fit yields: $a=2.582$, $b=3.642, c=1.484$ and $d=1.080$. The corresponding values for the $\mathrm{q}=0.5$ case are: $a=1.564, b=4.526, c=1.516$ and $d=1.037$.

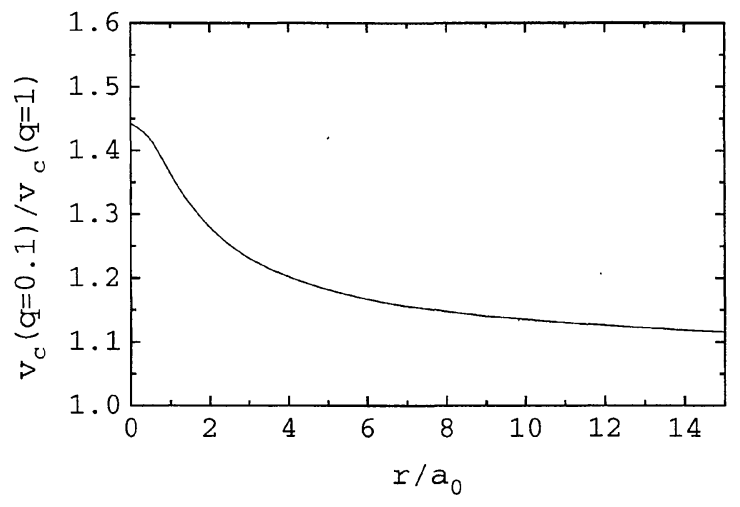

FIG. 15. The ratio of the rotation curve of a flattened mass distribution ( $q$ $=0.1)$ to that of a spherical distribution.

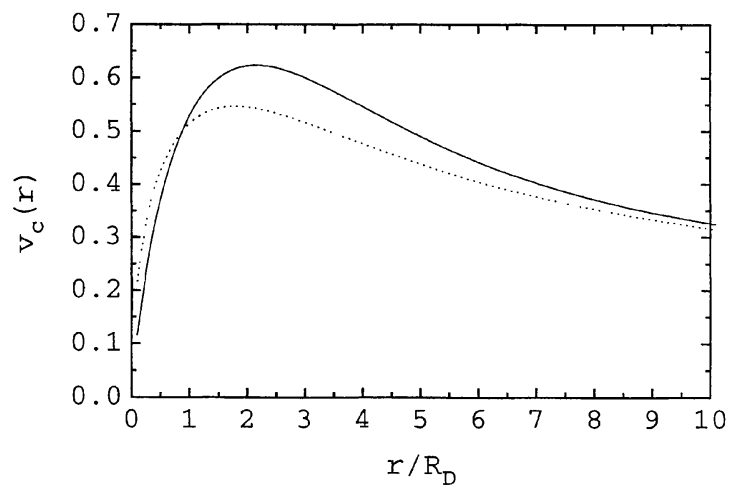

FIG. 16. Circular speed versus radius for an exponential disk (full curve) and a spherical body which has the same mass $M(r)$ interior to $r$ (adapted from Binney \& Tremaine).

Another important case is an infinitely thin mass distribution. For such a disk with a surface density $\sigma=\sigma_{0} e^{-r / R_{D}}$, the circular velocity is given by:

$$
v_{c}^{2}(r)=\pi G \sigma_{0} \frac{r^{2}}{R_{D}}\left[\mathrm{I}_{0}(\alpha) \mathrm{K}_{0}(\alpha)-\mathrm{I}_{1}(\alpha) \mathrm{K}_{1}(\alpha)\right],
$$

where $\alpha=r /\left(2 R_{D}\right)$ and $\mathrm{I}_{n}(\alpha)$ and $\mathrm{K}_{n}(\alpha)$ are modified Bessel functions.

Since all parameters in the above equations are known, we can directly calculate the expected rotation curve for an exponential disk. This was done in this investigation. For those who prefer not to deal with Bessel functions, we present a somewhat similar procedure as for the flattened ellipsoid case presented above: it is fairly easy to calculate a rotation curve for a hypothetical spherical mass distribution that has the same mass $M(r)$ interior to $r$ as the thin exponential disk, i.e., $M(r)=2 \pi \int_{0}^{r} \sigma_{0} e^{-r^{\prime} / R_{D}} r^{\prime} d r^{\prime}$. This erroneous curve is plotted in Fig. 16 (dotted line), in addition, the correct curve for an infinitesimally thin exponential disk (full line) is shown. Comparing these two curves gives us information on how we have to correct the "spherical calculation" to get the correct result.

We calculated the correction function $\Gamma(r)$ for this case, dividing the thin-disk curve by the spherical rotation curve.

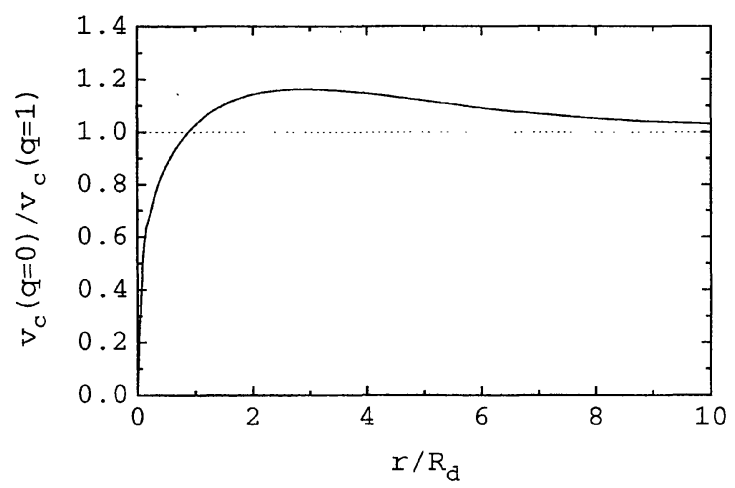

FIG. 17. The ratio of the rotation curve of an exponential disk to that of a spherical distribution. 
The result is plotted in Fig. 17 and a best fit yields: $\Gamma(r)=\left(a r^{b}-1\right) \exp (-c r)+1 \quad(a=1.046, \quad b=0.308, \quad c$ $=0.361)$. So, if we are dealing with an exponential disk, whose mass distribution is best approximated by an infinitesimally thin disk we can first calculate an approximation by assuming a spherical distribution and correct with the derived correction function to find the exact rotation curve.
Note that in the limit of large radii (in terms of scale lengths), the correction converges to a fairly constant value of 1.13 in the ellipsoidal and 1.05 in the infinitely flat case. For radii of a few scale lengths, however, the correction values are strongly varying with radius. Since this is an important regime for our studies of dwarf galaxies, the approach as outlined in this Appendix is necessary.

\section{REFERENCES}

Ashman, K. M. 1992, PASP, 104, 1109

Armandroff, T. E., Olszewski, E. W., \& Pryor, C. 1995, AJ, 110, 2131

Baars, J. W. M., Genzel, R., Pauliny-Toth, I. I. K., \& Witzel, A. 1977 , A\&A, 61, 99

Begeman, K. G. 1989, A\&A, 223, 47

Binney, J., \& Tremaine, S. 1987, Galactic Dynamics (Princeton University Press, Princeton, NJ), Chap. 2

Briggs, D. 1995, Ph.D. thesis, New Mexico Institute of Mining and Technology

Brinks, E. 1990, in Dynamics and Interaction of Galaxies, edited by R. Wielen (Springer, Heidelberg), p. 146

Brinks, E., \& Taylor, C. L. 1994, in ESO/OHP Conference and Workshop Proceedings No. 49, edited by G. Meylan and P. Prugniel, p. 263

Campos-Aguilar, A., Moles, M., \& Masegosa, J. 1993, AJ, 106, 1784

Carignan, C. 1985, ApJ, 299, 59

Carignan, C., \& Beaulieu, S. 1989, ApJ, 347, 760

Clark, B. G. 1980, A\&A, 89, 377

Côté, S., Carignan, C., \& Sancisi, R. 1991, AJ, 102, 904

Dickey, J. M., Hanson, M. M., \& Helou, G. 1990, ApJ, 352, 522

Erickson, L. K., Gottesman, S. T., \& Hunter, Jr., J. H. 1987, Nature, 325, 779

de Vaucouleurs, G., de Vaucouleurs, A., Corwin, H. G., Buta, R. J., Paturel,

G., \& Fouqué, P. 1991, Third Reference Catalogue of Bright Galaxies

(Springer, New York) (RC3)

Gerola, H., \& Seiden, P. E. 1978, ApJ, 223, 129

Högbom, J. A. 1974, A\&AS, 15, 417

Israel, F. P., Tacconi, L. J., \& Baas, F. 1995 A\&A, 295, 599

Jobin, M., \& Carignan, C. 1990, AJ, 100, 648

Jörsäter, S., \& van Moorsel, G. A. 1995, AJ, 110, 2037
Kamphuis, J. J. 1993, Ph.D. thesis, University of Groningen

Lake, G., Schommer, R. A., \& van Gorkom, J. H. 1990, AJ, 99, 547

Mateo, M. 1994, in ESO/OHP Conference and Workshop Proceedings No. 49, edited by G. Meylan and P. Prugniel, p. 309

Moles, M., García-Pelayo, J. M., del Río, G., \& Lahulla, F. 1987, A\&A, 186, 77

Mueller, M. W., \& Arnett, W. D. 1976, ApJ, 210, 670

Noguchi, M. 1988, A\&A, 203, 259

Ostriker, E. C., Huchra, J. P., Geller, M. J., \& Kurtz, M. J. 1988, AJ, 96, 1775

Persic, M., \& Salucci, P. 1991, ApJ, 368, 60

Puche, D., Westpfahl, D., Brinks, E., \& Roy, J.-R. 1992, AJ, 103, 1841

Sage, L. J., Salzer, J. J., Loose, H.-H., \& Henkel, C. 1992, A\&A, 265, 19

Takamiya, M., Kron, R. G., \& Kron, G. E. 1995, AJ, 110, 1083

Taylor, C. L., Brinks, E., \& Skillman, E. D. 1993, AJ, 105, 128

Taylor, C. L., Brinks, E., Pogge, R. W., \& Skillman, E. D. 1994, AJ, 107, 971

Taylor, C. L., Brinks, E., Grashuis, R. M., \& Skillman, E. D. 1995, ApJS, 99, 427

Taylor, C. L., Brinks, E., Grashuis, R. M., \& Skillman, E. D. 1996, ApJS, 102,189

Telles, E., \& Terlevich, R. 1995, MNRAS, 275, 1

Thuan, T. X., \& Martin, G. E. 1981, ApJ, 247, 823

Unger, S. W., Brinks, E., Laing, R. A., Tritton, K. P., Gray, P. M. 1988, Observers' Guide to the Isaac Newton Group Telescopes in La Palma van Moorsel, G. A. 1987, A\&A, 176, 13

Young, J. S., \& Knezek, P. M. 1989, ApJ, 347, L55

Zwicky, F., Herzog, E., Wild, P., Karpowicz, M., \& Kowal, C. T. 19611968, Catalogue of Galaxies and Clusters of Galaxies (California Institute of Technology, Pasadena) (CGCG) 